$$
\begin{aligned}
\text { NAL PROPOSAL No. } & 52 \\
\text { Correspondent: } & \begin{array}{l}
\text { H. Weisberg } \\
\text { Department of Physics } \\
\text { University of Pennsylvania } \\
\text { Philadelphia, Pa. 19104 }
\end{array} \\
\text { FTS/Off-net: } & \text { 215-597-3311 } \\
& 594-8141
\end{aligned}
$$

\title{
A PROPOSAL TO STUDY PARTICLE PRODUCTION SPECTRA AND MULTIPLICITIES IN HIGH ENERGY HADRON-HADRON COLLISIONS, AND FOR A BEAM SURVEY AND QUARK SEARCH
}

E. W. Beier, D. I. Kreinick, H. Weisberg University of Pennsylvania 


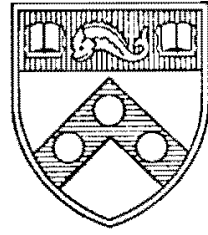

The College

\title{
UNIVERSITY Of PENNSYLVANIA
}

PHILADELPHIA 19104

Department of Physics

\author{
A PROPOSAL \\ TO STUDY PARTICLE PRODUCTION \\ SPECTRA AND MULTIPLICITIES \\ IN HIGH ENERGY HADRON-HADRON \\ COLLISIONS, AND FOR A \\ BEAM SURVEY AND QUARK SEARCH
}

We propose an experimental study at the new 500 Gev accelerator of the differential cross-section for particle production in hadron-hadron collisions. The projectile, and the observed single particle, will range over all combinations of positive and negative $\pi, K$ and $p$, with momenta extending up to the highest available. Enough of the secondary particle momentum range will be covered to permit us to determine by integration the multiplicity of the produced particle.

Single particles will be detected in a simple spectrometer consisting of wire chambers and a small bending magnet. The configuration of the spectrometer components will be variable so that the overall spectrometer length can be kept proportional to the secondary momentum. The momentum resolution $8 \mathrm{P} / \mathrm{P}= \pm 0.8 \%$ and the invariant phase space acceptance $\mathrm{P}^{2} \mathrm{~d} \Omega \mathrm{dP} / \mathrm{E}=1.3 \times 10^{-3}(\mathrm{Gev} / \mathrm{c})^{2}$ will then be the same at all momenta. Particle identification will be by means of threshold Čerenkov counters, with 104: 1 rejection up to at least $250 \mathrm{Gev} / \mathrm{c}$.

Our experimental arrangement is thought to be simple and yet powerful, and we propose its use initially with incident protons and a nuclear target for a beam survey and quark search. Subsequent measurements will be carried out with a hydrogen target in a high intensity secondary beam.

Experimenters: Eugene W. Beier, David L. Kreinick and Howard Weisberg

Date: $\quad$ June 15,1970

Correspondent: Howard Weisberg 
$\underline{\text { TABLE }}$ OF $\underline{\text { CONTENTS }}$

2. Theories of inelastic hadron-hadron scattering

3. The experimental arrangement

4. Kinematics

5. Sensitivity

6. Backgrounds and maximum beam intensity 28

7. Particle identification

8. System acceptance, resolution and other performance factors

9. Beam survey and quark search

10. Running time

11. Beam requirements

12. Equipment requirements and manpower 


\section{INTRODUCTION}

Multiparticle production plays a dominating role in hadron-hadron scattering at high energies. The detailed experimental study of the multiparticle channels becomes exceedingly difficult as the energy increases, especially because of the problem of missing neutrals. Furthermore, even if one had detailed information, its theoretical understanding would be difficult because of the problem of organizing the vast amount of data that would be involved. These considerations lead one to hope that, by studying simple features of high energy scattering, some groundwork for theoretical understanding can be laid. Feynman ${ }^{1}$ and others have emphasized this line of argument.

The simplest features of inelastic scattering are:

(1) total cross sections

(2) multiplicities

(3) single particle momentum distributions

(4) two particle correlation functions

Each of these can be studied as a function of energy and particle type. our experiment is intended to be a rather comprehensive first look at

(2) and (3) in a newly accessible energy range.

Specifically we propose to measure the differential cross section for the production of particle $c$ in the reaction $a b \rightarrow c d$,

$$
\frac{d \sigma}{d^{3} \underline{p} *}(a b-c) \equiv \sum_{d} \frac{d \sigma}{d^{3} \underline{p} *} \quad(a b-c d)
$$

where $a, c=p, \bar{p}, \pi^{ \pm}$, and $k^{ \pm}, b=p, d$ ranges over all available states and is not detected in our experiment, and $\underline{\mathrm{p}} *$ is the $\mathrm{cm}$ 
momentum of $c$.

We sha11 measure the dependence of these distributions on longitudinal c.m. momentum $\mathrm{P}_{\|} *$, transverse momentum $\underline{\mathrm{P}}_{\mathrm{T}}$, incoming c.m. momentum $\mathrm{P}_{\mathrm{a}}^{*}$ and on the identities of particles $\mathrm{a}$ and $\mathrm{c}$ Furthermore our measurements shall cover a wide enough range of $\underline{p} *$ to permit us to determine the average multiplicity $\bar{n}$ of particle $c$ from the integral

$$
\bar{n} \sigma=\int \frac{d \sigma}{d^{3} \underline{p} *} \quad d^{3} \underline{p} *
$$

where $\sigma$ is the total cross section, which will be known from other experiments.

This experimental approach will be particularly fruitful if it should turn out that multiparticle production has some of the features of a rather random, incoherent process, in the spirit of the original Fermi statistical model ${ }^{2}$, or of current ideas of limiting fragmentation $^{3}$ and partons ${ }^{1,4}$, as discussed below in Section 2 .

Recently there has been much interest in studies of the process of inelastic electron scattering, because of the observation that, as the momentum transfer increases, the "bumps" die away while the "background" holds up, and that an apparent scaling law holds. 5 For hadron-hadron scattering, the analogous facts are that as the energy goes up, cross sections for specific channels fall off with Regge $s^{\alpha}$ behavior, and that there is an apparent tendency to approach limiting distributions for the remaining, largely inelastic, processes. These facts suggest an analogous interest in hadron-hadron inelastic 
scattering studies of the type proposed here.

Finally, we believe that there is merit in studying single particle production spectra that is independent of any particular theoretical ideas that one may be testing. These distributions are simple, basic, and easily acessible experimentally. The richness of the range of information available suggests that the data may well turn out to have significance that can only be seen a posteriori.

This type of experiment seems to have been somewhat neglected in the past, with a few notable exceptions of particle production in pp collisions ${ }^{6}$ and one recent result on longitudinal moments of negatives in $\pi^{-} \mathrm{p}$ collisions ${ }^{7}$.

In Section 2 we review theoretical ideas about inelastic hadronhadron scattering. In Section 3 we describe the experimental apparatus, a spectrometer with length proportional to the momentum measured. This variable length spectrometer enables us to obtain a momentum resolution and acceptance which are independent of momentum and it covers the entire kinematic range of the distribution we are mea suring.

In Section 4, we discuss the kinematics of particle production, to determine what range of laboratory momenta and angles must be covered. In Section 5, we give a simple estimate of the counting rates, and a determination of the momentum and angle acceptance needed to achieve a given experimental sensitivity. 
In our experimental design, there is a limit to the maximum beam intensity that can be used. This limit is essentially set by the requirement that the singles rates (counting rate integrated over all momenta) in the counters and wire chambers not be too great. In section 6 we use the counting rate analys is of Section 5 to estimate the maximum beam rate that we can handle; this limit turns out to be independent of the spectrometer length, and is around $10^{8}$ particles per second.

Particle identification is discussed in Section 7. Here again a variable length design is used. The needed threshold Čerenkov counter length is obtained by bolting various lengths of tubing together, with a phototube and mirror module on the end. The total mass of radiator is the same at all momenta. However, the length needed goes as the square of the momentum, so our simple scaling property breaks down and there is a momentum at which the entire available spectrometer length is filled with Čerenkov counters .

The detailed performance of the spectrometer is discussed in section 8. In particular, the dependence of the acceptance on momentum and the effect of multiple scattering on the momentum resolution are presented. Also the momentum range of the threshold Cerenkov counters is compared to the momentum bite of the spectrometer.

In Section 9 we propose that our experiment be set up initially with a proton beam to carry out a beam survey and quark search. Section 10 gives an estimate of the running time needed, Section 11 discusses beam requirements and Section 12 discusses equipment and manpower. 


\section{THEORIES OF HIGH ENERGY HADRON-HADRON INELASTIC SCATTERING}

In the Fermi statistical mode ${ }^{2}$, the energy of the incident particles is dumped into a small region whose size is of the order of the incident particle size; this energy then escapes, in the form of hadronic matter, with equal probability into all available channels. It is well known that this model seriously disagrees with observation. In particular it fails to account for the fact that transverse momenta are always limited to small values, even at high bombarding energy. Nonetheless the model, which was formulated before there was quantitative data on particle production, retains its appea1. It provides one with the simple picture of the collision of two extended blobs of hadronic matter with rather well defined sizes, and to this day it provides bump hunting experimental physicists with a convenient parameterization for the vast expanses of inelastic background which, at high energies; underlie the progressively tinier and tinier bumps that they find.

Hagedorn ${ }^{8}$ has modified the statistical model to get agreement with existing observations, by invoking a maximum temperature for nuclear matter and by considering particles emanating from two outgoing "firebal1s" associated with the incoming hadrons.

Yang $^{3}$ has emphasized the picture of hadrons as extended objects that are rather transparent to each other. This picture leads to the hypothesis of limiting fragmentation, by the following argument. Viewed in the rest system of the target, the projectile is a pancake 
shaped object passing by with essentially the velocity of light. At high energies, some or all of the matter in the target might then be expected to emerge in a way that, at high bombarding energy, no longer depends on the energy. Similarly there would be emerging particles that are fragments of the projectile, and have limiting momentum distributions in the projectile rest frame. Existing experimental data tend to support this hypothesis. The increasing multiplicity at high energies would then correspond, at least in part, to more and more of the limiting distribution becoming kinematically accessible.

Feynman $^{2}$ reaches essentially the same conclusion about limiting distributions by arguments based on the picture that hadrons are made up of point-like constituents (partons) whose interactions are similar to those of simple field theory. These arguments are conveniently stated in frames in which the incoming particles have large momentum, and in particular in the c.m. frame. The incoming hadrons are made up virtually of constituents, each characterized by $x$, its fraction of the incoming longitudinal momentum and by $P_{T}$, its transverse momentum, which is hypothesized to be small. Simple quantum mechanical arguments then suggest that in the collision only patrons of "wee" $\mathrm{x}$ - those having longitudinal momentum in the c.m. of $s 1 \mathrm{Gev} / \mathrm{c}$, can be exchanged. In that case, the momentum distribution of the outgoing constituents and hence, after appropriate final state interactions, of the outgoing particles observed in the $1 \mathrm{ab}$, will reflect that of the constituents coming in. 
The limiting fragmentation idea translates for the single particle momentum distributions in the c.m. into the statement that

$$
\frac{\mathrm{d} \sigma}{\mathrm{dxd}^{2} \mathrm{p}_{\mathrm{T}}}=\mathrm{f}\left(\mathrm{x}, \mathrm{p}_{\mathrm{T}}\right)
$$

where $f$ does not depend on the incident energy. Particles of definite negative $x$ correspond, by Lorentz transformation, to particles of definite momentum in the target frame, while particles of definite positive $\mathrm{x}$ correspond to particles of definite momentum in the projectile rest frame, in the limit of high incoming energies.

One then will have target independence: the momentum distribution of the fragments of the projectile $(x>0)$ will depend only on the identity of the projectile and not of the target, and conversely for the secondaries with $x<0$. A further prediction is that transverse momenta will be limited, independent of energy.

A hint that the parton model may have some validity comes from inelastic electron scattering, which behavior can be explained by elastic scattering of the electrons from point partons having a fraction $x=Q^{2} / 2 M \nu$ of the total incoming proton momentum. ${ }^{5}$ In fact if the parton model is correct, the observed cross section for a given $\mathrm{x}$ is a measure of the probability of finding a parton with longitudinal fraction $x$ in the incoming hadron. According to the observed cross sections, this probability goes as $1 / x$.

For hadron-hadron collisions, the increase with energy of the multiplicity comes from the fact that more and more of the region of 
sma11 $\mathrm{x}$ becomes available. At any finite energy the $1 / \mathrm{x}$ distribution would extend only down to the "wee" region rather than to zero. The multiplicity is equal to the integral of the longitudinal momentum distribution over a11 $\mathrm{x}$, divided by the total cross section. If the total cross section is constant with energy, then by integrating $1 / \mathrm{x}$ down to $\mathrm{x}=[1 \mathrm{Gev} /$ incident energy], we find a logarithmic dependence of the multiplicity on energy.

A number of the experimental predictions mentioned above, such as limited transverse momentum and inelasticities, target independence, logarithmic increase of multiplicity with energy and $1 / x$ longitudinal momentum dependence for small $\mathrm{x}$, also come from multiperipheral and multi-Regge models. ${ }^{9}$

Figure 1 shows some experimental information on single particle longitudinal momentum distributions. The data on $p p-p$ and $p p-\pi^{-}$ were obtained by numerical integration of the strong-focussing single-arm spectrometer data of Allaby et $a 1^{6}$. The other two curves were obtained from new bubble chamber data of Elbert and Erwin ${ }^{7}$ on the longitudinal momentum dependence of negative particles from $25 \mathrm{Gev} \pi^{-} \mathrm{p}$ collisions, in which all negative particles are assumed to be $\pi^{-}$s. Their data for positive longitudinal momentum are presented as $\pi^{-} \mathrm{p} \rightarrow \pi^{-}$while their data for negative longitudinal momentum have been reflected about $\mathrm{P}_{n}=0$ and labeled $\mathrm{p}^{-}{ }^{-} \pi^{-}$. The measured values of $\frac{\mathrm{d}_{\sigma}}{\mathrm{dx}}$ have in each case been multiplied by a factor of $\frac{\mathrm{x}}{\sigma}$ where the $\sigma$ factor is included to get a dimensionless quantity, and 


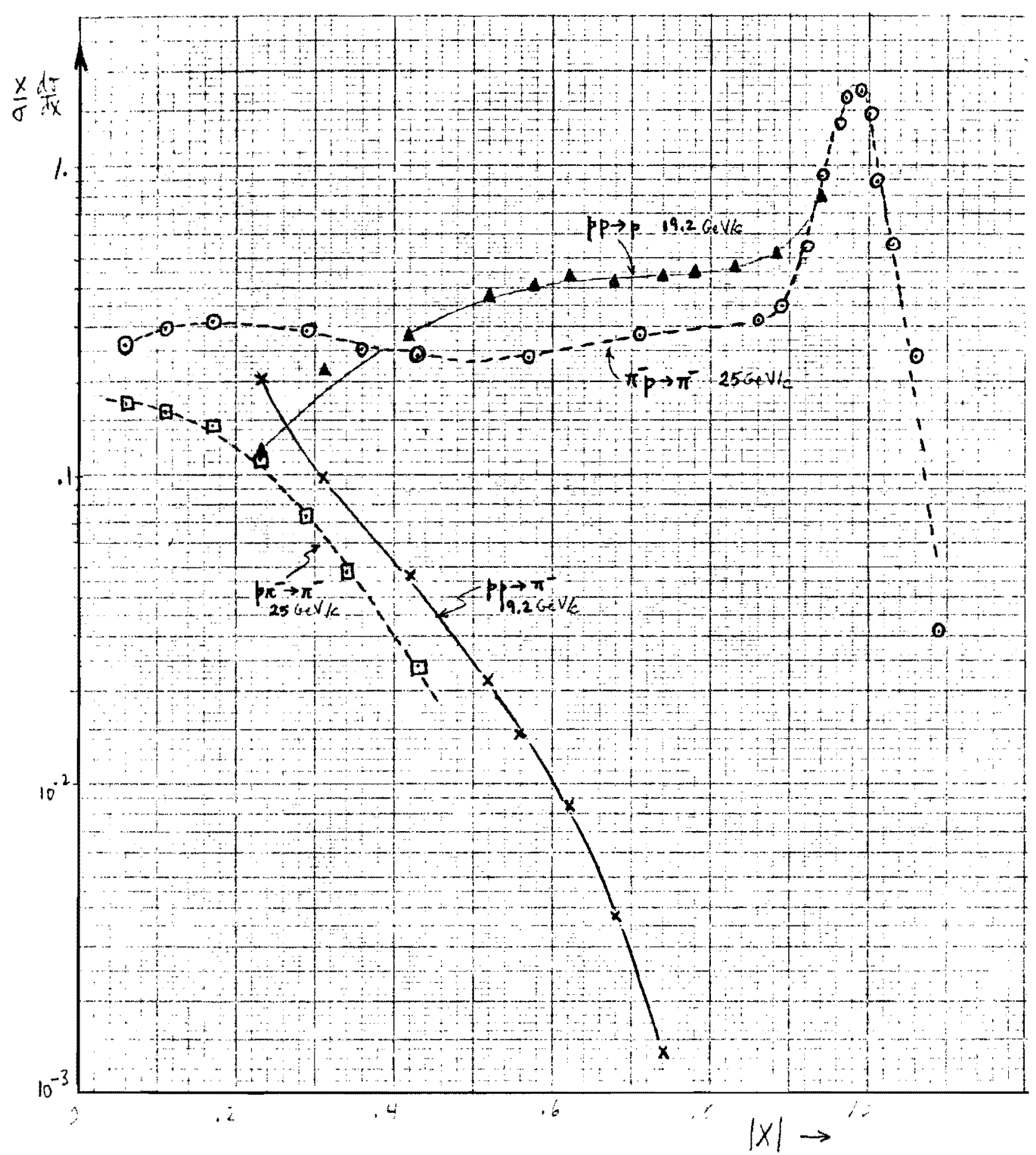

FIGURE 1. Experimentally observed values of $\frac{1}{\sigma} \times \frac{\mathrm{d} \sigma}{\mathrm{dx}}$. The curves for $\pi^{-} \mathrm{p}-\pi^{-}$and $\mathrm{p} \pi^{-} \rightarrow \pi^{-}$were obtained from the data of ${ }^{\mathrm{dx}}$ Reference 7 as discussed in the text. The $\mathrm{p}^{-}{ }^{-} \pi^{-}$curve was obtained from the $\pi^{-} p \rightarrow \pi^{-}$data for $x<0$ by reflection about $x=0$. The curves for $p p \rightarrow p$ and $\mathrm{pp}^{-4} \pi^{-}$were obtained from the data of Reference 6 by numerical integration over the transverse momenta. 
the $\mathrm{x}$ factor is included so that a $1 / \mathrm{x}$ longitudinal momentum dependence would appear as a tendency for the data to approach a constant

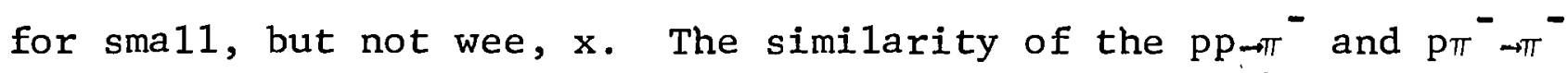
curves suggests that some sort of target independence may indeed hold. 
3. THE EXPERIMENTAL ARRANGEMENT.

Our experimental design is based on the following ideas:

(1) Transverse momenta in hadron-hadron collisions are 1 imited to $\lesssim 1 \mathrm{Gev} / \mathrm{c}$. Therefore in order to cover the entire range of $x$ from -1 to +1 , we need to cover essential1y two regions of laboratory momentum and angle, a smal1 angle "forward region" covering a wide momentum range, and a "backward" region covering al1 1aboratory angles but with momentum limited to $\lesssim 1 \mathrm{Gev} / \mathrm{c}$.

(2) A momentum resolution of the order of $1 \%$ is more than adequate for the phenomena we wish to study. Since with careful design the transverse momentum from multiple scattering can be kept to a few Mev/c, the analyzing magnet need only have a transverse momentum impulse of a few hundreds of Mev/c.

(3) The acceptance $\mathrm{P}^{2} \mathrm{~d} \Omega \frac{\mathrm{dP}}{\mathrm{E}}$ in invariant phase space should be rough1y constant over the entire range covered.

(4) The instantaneous beam rate in a secondary beam at NAL will be $\sim 10^{8}$ particles per second. Since we can therefore use detectors which view the target directly, we do not need to use a strong-focussing spectrometer. This situation is unlike the one at SLAC, where the instantaneous beam rate in the external beam is $\sim 10^{9}$ times greater. Based on these considerations, we have designed a variable 
configuration single-arm spectrometer, consisting of wire chambers and a small magnet. The "forward" region is covered by a sequence of configurations of different lengths, ranging from 200 meters down to 3.1 meters, covering momenta from $160 \mathrm{Gev} / \mathrm{c}$ down to $1.25 \mathrm{Gev} / \mathrm{c}$, at transverse momenta from $0.13 \mathrm{Gev} / \mathrm{c}$ up to $3.5 \mathrm{Gev} / \mathrm{c}$. The "backward" region is covered by a small fixed length spectrometer pivoted around the target.

It is expected that each configuration changeover can be carried out in a few hours; simple optical techniques will be used for alignment. The schedule of data taking will be arranged so that we take a complete set of data at each spectrometer configuration before changing lengths; there will be 8 configurations.

Figure $2 \mathrm{a}$ is a sketch of the layout for the "forward" data taking. The beam, which contains an unseparated mixture of pions, kaons and protons or antiprotons, is incident on a liquid hydrogen target. Cerenkov counters in the beam (not shown) identify the incident particles. The unscattered beam travels to a distant shielded beam dump. Scattered particles are detected, and their trajectories determined, in wire chambers $W_{1,2,3}$. Momentum analysis is provided by magnet $M_{1}$, and the trajectories after the magnet are determined by wire chambers $W_{4,5,6}$. Threshold Cerenkov counters $\check{\mathrm{C}}_{1}, \stackrel{\check{C}}{\mathrm{C}}_{2}$, and $\check{\mathrm{C}}_{3}$ determine whether the scattered particle is a proton, a kaon or a light particle (pion, 


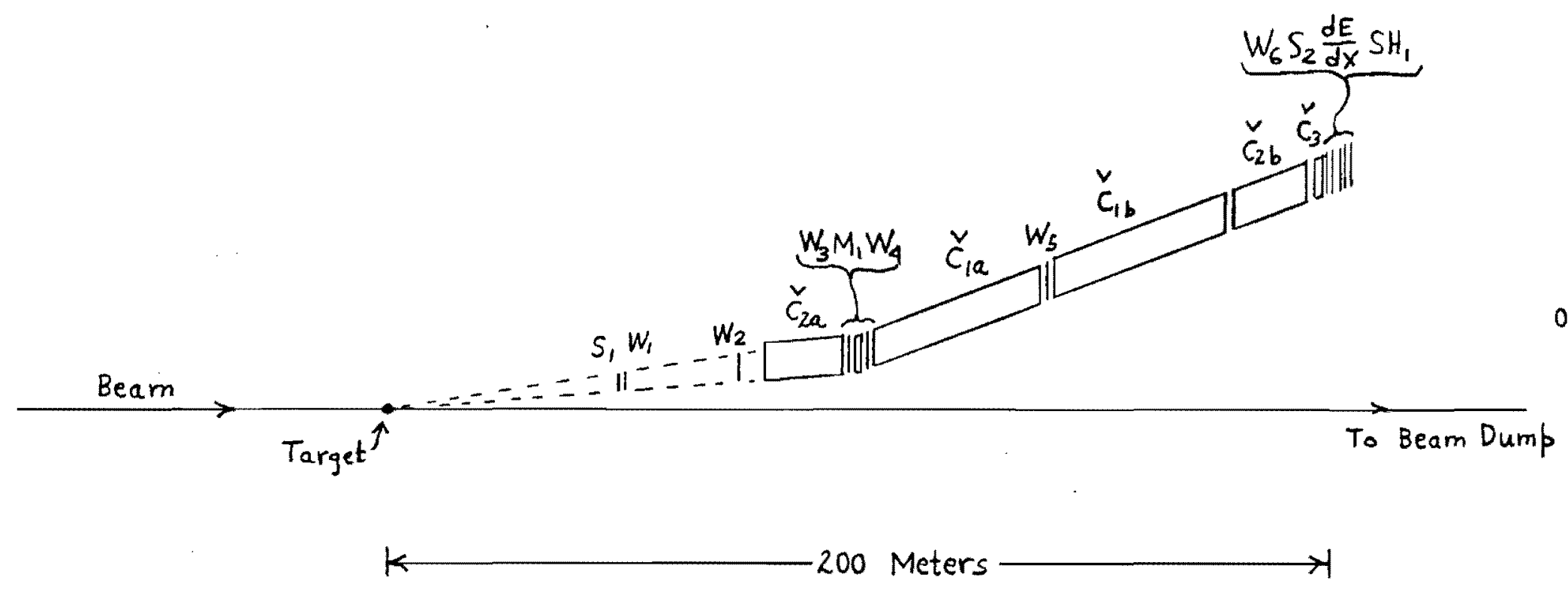

Figure 2a. A sketch of the layout of the "forward" spectrometer configuration, as seen from above. The dimensions shown are for the momentum band $80-160 \mathrm{Gev} / \mathrm{c}$. The scale for any other momentum band is obtained by scaling the longitudinal distances 1inearly with momentum. 
muon or electron). The event trigger is basically a coincidence between scintillation counters $s_{1}$ and $s_{2}$ and a Čerenkov counter. Energy loss counters ("dE/dX" in the figure) identify particles of non-integral charge, quarks in particular; shower counter $\mathrm{SH}_{1}$ identifies electrons. Muons are distinguished from pions by the requirement that they traverse several interaction lengths of matter.

The needed coverage in transverse momentum is obtained by moving the spectrometer components on rails transverse to the beam. The motion will be remote controlled so that changes can be effected in a very short time. Since the scattering angle is sma11, transverse motion is just as good as motion along arcs centered on the target. In order to reach a transverse momentum of $3.5 \mathrm{Gev} / \mathrm{c}$, the experimental area has to extend out to one side of beam center line a distance of

$$
\frac{3.5 \mathrm{Gev} / \mathrm{c}}{160 \mathrm{Gev} / \mathrm{c}} \times 200 \text { meters }-0.5 \text { Meters }=3.9 \text { meters }
$$

The saving of 0.5 meters comes from bending toward rather than away from the beam line when we are at large angles.

There is an alternative way to vary the scattering angle, which is to use deflecting magnets and keep the spectrometer fixed. A magnet just downstream of the target, bending in the vertical plane, can allow us to vary the production angle of the detected particles. A rather large magnet is required ( 6 meters 
to reach $\mathrm{P}_{\mathrm{T}}=3.5 \mathrm{Gev} / \mathrm{c}$ ). For a number of reasons we prefer the transverse motion method, although the bending magnet method might be attractive in an initial beam survey phase in the proton beam tunne1 as discussed in Section 9 .

To cover the "backward" region, we use a fixed length spectrometer pivoted around the target. A drawing of the layout is given in Figure $2 \mathrm{~b}$. The magnet $\mathrm{M}_{1}$ is the same as is used in the "forward" configuration, as are the entrance chambers, readout electronics, software, etc. Special chambers, somewhat larger than the ones for the "forward" configuration, are used after the magnet to make up for the loss of aperture arising from the fact that the magnet length is now a significant fraction of the total spectrometer length. A Berkeley-type $13 \times 24 \mathrm{C}$ magnet is shown for $M_{1}$. At full excitation the momentum band covered is 0.5 to $1.0 \mathrm{Gev} / \mathrm{c}$; lower momenta are obtained by reducing the excitation. The laboratory angle coverage extends from 25 to 155 degrees . 
(a)

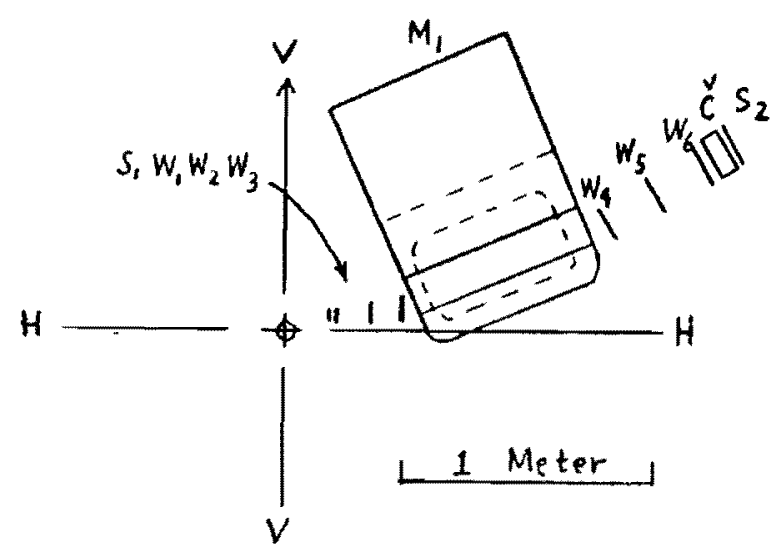

(b)

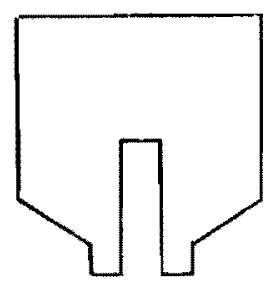

Figure 2b. The low momemtum ("backward") spectrometer configuration: (a) side view; (b) section through magnet. The spectrometer is shown at $90^{\circ}$ laboratory angle, with the beam coming out of the paper. $\mathrm{HH}$ is the horizontal plane through the target. The entire spectrometer assembly is bolted together and pivots about the vertical axis VV through the target. Laboratory angles of 25 to 155 degrees can be reached. 


\section{KINEMATICS}

In this section we discuss some kinematical features of inelastic scattering at high energies. In particular we exhibit the limiting forms that the relation between laboratory and $\mathrm{c} . \mathrm{m}$. quantities take under typical high energy conditions, when the longitudinal momentum of a particle is large compared to its transverse momentum and mass. The experiment is designed to exploit these relationships.

Let the laboratory 4-momenta of particles a, b and $c$ in the reaction $a b \rightarrow c d$ to be given by

$$
\begin{aligned}
& \mathrm{p}_{\mathrm{a}}=\left(\mathrm{E}_{\mathrm{a}}, \mathrm{P}_{\mathrm{a}}, 0\right) \\
& \mathrm{p}_{\mathrm{b}}=(\mathrm{M}, 0,0) \\
& \mathrm{p}_{\mathrm{c}}=\left(\mathrm{E}, \mathrm{P}_{\|}, \mathrm{P}_{\mathrm{T}}\right)
\end{aligned}
$$

where the components given are the total energy, longitudinal momentum and transverse momentum of each particle, and the masses are $\mathrm{m}_{\mathrm{a}}=\mathrm{m}, \mathrm{m}_{\mathrm{b}}=\mathrm{M}$ and $\mathrm{m}_{\mathrm{c}}=\mu$. The corresponding center of mass quantities are

$$
\begin{aligned}
& \mathrm{P}_{\mathrm{a}}^{*}=\left(\mathrm{E}_{\mathrm{a}}^{*}, \mathrm{P}_{\mathrm{a}}^{*}, 0\right) \\
& \mathrm{P}_{\mathrm{b}}^{*}=\left(\mathrm{E}_{\mathrm{b}} *,-\mathrm{P}_{\mathrm{a}} *, 0\right) \\
& \mathrm{P}_{\mathrm{c}}{ }^{*}=\left(\mathrm{E}^{*}, \mathrm{P}_{\|} *, \mathrm{P}_{\mathrm{T}}\right)
\end{aligned}
$$

Setting

$$
\mathrm{s}=\left(\mathrm{p}_{\mathrm{a}}+\mathrm{p}_{\mathrm{b}}\right)^{2}=\mathrm{m}^{2}+\mathrm{M}^{2}+2 \mathrm{ME} \mathrm{E}_{\mathrm{a}}=\left(\mathrm{E}_{\mathrm{a}} *+\mathrm{E}_{\mathrm{b}} *\right)^{2}
$$


we have

$$
\mathrm{P}_{\mathrm{a}} *=\mathrm{P}_{\mathrm{a}} \frac{\mathrm{M}}{\sqrt{\mathrm{s}}}
$$

or, at high energies

$$
P_{a} * \simeq \sqrt{\frac{M P}{2}}
$$

For the laboratory longitudinal momentum we have

$$
\mathrm{P}_{\|}=\frac{M P_{a}}{s}\left[x\left(E_{a}+M\right)+\sqrt{\left.x^{2} P_{a}^{2}+\frac{\mu^{2}+P_{T}^{2}}{M^{2}} s\right]}\right.
$$

where we have introduced the center of mass longitudinal

fraction

$$
\mathrm{x}=\frac{\mathrm{P}_{\|}}{\mathrm{P}_{\mathrm{a}}{ }^{*}}
$$

For fixed $x$, the high energy limit of this is

$$
P_{\|}=\frac{1}{2}\left[x P_{a}+x M+|x| P_{a} \sqrt{\left.1+\frac{x^{2}}{x^{2}}\right]}\right.
$$

where

$$
x_{0}=\frac{\sqrt{\mu^{2}+P_{T}^{2}}}{P_{a}^{*}}
$$

The quantity $x_{0}$ corresponds in size to what Feynman calls "wee" (as opposed to"sma11") $\mathrm{x}$ : wee $\mathrm{x}$ means $|\mathrm{x}| \leq \frac{1 \mathrm{Gev} / \mathrm{c}}{\mathrm{P}_{\mathrm{a}}^{\mathrm{x}}}$, while small $\mathrm{x}$ means simply $\mathrm{x}<<1$.

For positive $\mathrm{x}$ we have the following simple limiting relation between $\mathrm{P}_{\|}$and $\mathrm{x}$ :

$$
\mathrm{P}_{\|} \approx \mathrm{xP}_{\mathrm{a}} \text {, for } \mathrm{x}_{\mathrm{o}} \ll \mathrm{x} \leq 1
$$

In other words, the longitudinal fraction in the lab is the same as the longitudinal fraction in the center of mass, and 
is independent of masses.

For negative $x$ we have a different kind of limiting behavior:

$$
\mathrm{P}_{\| 1}=\frac{\mathrm{M}}{2} \mathrm{x}-\frac{\mu^{2}+\mathrm{P}_{\mathrm{T}}^{2}}{2 \mathrm{M}} \frac{1}{\mathrm{x}} \text {, for }-1 \leq \mathrm{x} \ll-\mathrm{x}_{\mathrm{o}}
$$

In other words finite $P_{11}$ in the lab corresponds to a definite value of $\mathrm{x}$ in the center of mass. The scale factor for the dependence is set by the target mass $M$ and the produced particle mass $\mu$, without any dependence on the incident particle mass $\mathrm{m}$.

The values of $P_{\|}$for positive and negative $x$ join smoothly on to each other in the region $|x| \leq x_{0}$, passing through the value

$$
P_{\|}=x_{o} P_{a} \sqrt{\frac{M P a}{2 s}} \approx \frac{1}{2} x_{o} P_{a} \text {, for } x=0
$$

The behavior of $\mathrm{P}_{\|}$is illustrated in Figure 3, which shows calculated values of laboratory longitudinal momenta for protons, pions and kaons produced by $30 \mathrm{Gev}$ and $200 \mathrm{Gev}$ incident particles on a proton target. The curves shown are for incident protons and for zero transverse momenta, but the same curves, to within plotting errors, hold for incident pions or kaons, and for transverse momenta $\leq 1 \mathrm{Gev} / \mathrm{c}$. 


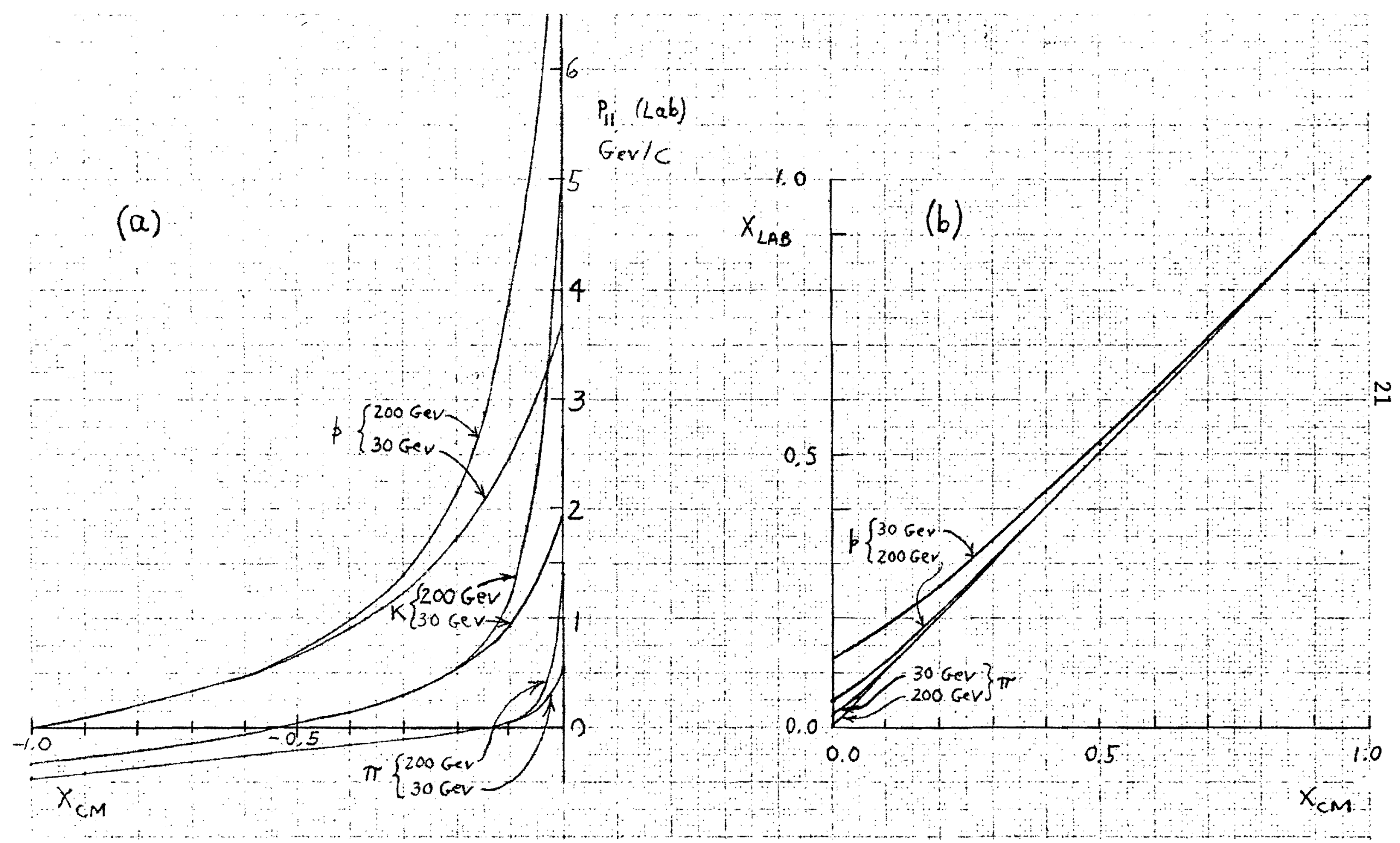

FIGURE 3. Relation between $1 \mathrm{ab}$ and c.m. longitudinal momentum, for zero transverse momentum. (a) The laboratory momentum $P_{11}$ is plotted against $x$, for $x<0$, for protons, kaons and pions from $\mathrm{pp}-\mathrm{p}, \mathrm{pp}-\mathrm{K}$ and $\mathrm{pp} \rightarrow \pi$ at 30 and $200 \mathrm{Gev}$. (b) The quantity $x_{A B}=P_{\|} / P$ is plotted against $x$, for $x>0$ for protons and pions from $\mathrm{pp} \rightarrow \mathrm{p}$ and $\mathrm{pp} \cdots \mathrm{H}_{\text {at }}$ at 30 and $200 \mathrm{Gev}$. 
These curves have the following implications for an experimental design. We are interested in covering the entire region of $0 \leq \mathrm{P}_{\mathrm{T}} \lesssim 1 \mathrm{Gev} / \mathrm{c}$ and $-1<\mathrm{x}<1$, including $\mathrm{a}$ study of the behavior around $\mathrm{x}=0$. Some information is also useful for larger $P_{T}$, over at least part of the range of $x$. Therefore in the laboratory one should ideally cover the following range of momenta: (a) from the full beam momentum down to $\sim 1 \mathrm{Gev} / \mathrm{c}$ at small forward angles corresponding to transverse momenta of $\lesssim 1 \mathrm{Gev}$; and (b) from $\sim 1 \mathrm{Gev} / \mathrm{c}$ down to zero over the entire range of $1 \mathrm{ab}$ angles from 0 to $180^{\circ}$. 


\section{SENS ITIVITY}

We shall parameterize the differential cross section in a simple way suggested by some of the theoretical conjectures in Section 2 to get an estimate of the event rate in terms of the laboratory solid angle and momentum acceptance of our detector. In doing so we are by no means tying the success of our experimental design to the validity of the theoretical conjectures; we are simply using these coniectures as a convenient way to incorporate presently known features of inelastic hadron behavior in the extrapolation to a new energy region. Our experimental design is rather different from what it would be if we had to approach the problem blindly, in which case we would be forced into a considerably more cumbersome and less flexible design.

Without loss of generality we write the differential cross section for $a b \rightarrow c$ as

$$
\frac{\mathrm{d} \sigma}{\mathrm{dxd^{2 } \mathrm { P } _ { \mathrm { T } }}}=\frac{\sigma_{0}}{\Delta^{2}} \frac{\mathrm{P}_{\mathrm{a}}^{*}}{\mathrm{E}^{*}} \mathrm{f}\left(\mathrm{P}_{\mathrm{a}}^{*}, \mathrm{x}, \mathrm{P}_{\mathrm{T}}\right)
$$

where $\sigma_{o}$ is the total cross-section for scattering of $a$ on $b$, $f\left(P{ }_{a}^{*}, x, P_{T}\right)$ is a dimensionless function, $\Delta$ is a constant with the dimensions of momentum and, as in section $4, E^{*}$ is the c.m. energy of particle c. If limiting fragmentation holds, then f will be a function of $x$ and $P_{T}$ only, and not of the incident momentum $P_{a}$. This fact suggests that, in our estimate of the counting rate at high energies, we use the values of $f$ determined in experiments at 
existing energies. Furthermore, it appears from data at present energies that $f$ roughly factors into the product of a function of $\mathrm{x}$ and a function of $\mathrm{P}_{\mathrm{T}}$. For our estimates it will be convenient to assume that this factorization holds, although this assumption is by no means critical. Thus we are led to the approximate form

$$
\frac{\mathrm{d} \sigma}{\mathrm{dxd^{2 } P _ { T }}}=\sigma_{0} \frac{\mathrm{P}_{\mathrm{a}}^{*}}{\mathrm{E}^{*}} \mathrm{~F}(\mathrm{x}) \frac{1}{\Delta^{2}} \mathrm{G}\left(\mathrm{P}_{\mathrm{T}}\right)
$$

where $F(x)$ and $G\left(P_{T}\right)$ are dimensionless functions. By definition $G$ is normalized to satisfy

$$
\iint d^{2} P_{T} \frac{1}{\Delta^{2}} G\left(P_{T}\right)=1
$$

From the general nature of the transverse momentum distribution in hadron-hadron scattering we know that, for a value of the constant $\Delta$ of the order of $0.4 \mathrm{Gev}$ the function $\mathrm{G}$ will be of the order of unity for small $\mathrm{P}_{\mathrm{T}}$, while it will fall off rapidly below unity for $\mathrm{P}_{\mathrm{T}}$ large compared to $\Delta$. (An example for which this behavior holds is $\left.\frac{1}{\Delta^{2}} G\left(P_{T}\right)=\frac{1}{.4^{2}} e^{-P_{T} / 16}\right)$.

As for the longitudinal dependence, we have that, for $\mathrm{x}$ not "wee",

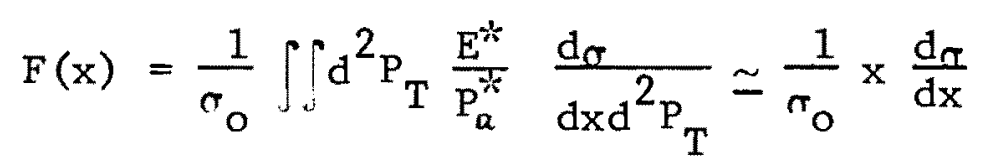

The data of figure 1 suggest that, depending on the identity of particles $a, b$ and $c, F(x)$ may be of order unity, or it may be smaller 
than this by many orders of magnitude, but that it does not exceed a value of the order of unity for $x$ not "wee". As for the "wee" region of $x$, there are very few data, but the results of Elbert and Erwin ${ }^{7}$ for $\pi^{-} \rightarrow^{-}$are consistent with $F(x)$ being we11 behaved and of order unity for "wee" $\mathrm{x}$.

We have now parameterized the differential cross-section in terms of dimensionless functions $F$ and $G$ which take on maximum values of order unity in some regions of phase space, while falling off to very sma11 values in other regions.

In the remainder of this section we use this parameterization for two purposes: to ensure that the acceptance of our apparatus is not so large that there is a significant probability of two particles from a single event being accepted; and to determine our sensitivity to smal1 cross-sections (values of $F(x) G\left(P_{T}\right)$ sma11 compared to unity). In Section 6 we sha11 use the parameterization again to ensure that, when our apparatus is set to respond to regions of phase space where the cross-section is sma11, there is no background contamination from leakthrough of events from regions where the cross-section is large.

First we need to transform our expression for the differential cross-section to 1aboratory quantities. This transformation is simple; if the laboratory solid angle is $\mathrm{d} \Omega$ and the laboratory momentum acceptance is $\mathrm{dP}$, we find 


$$
\frac{\mathrm{d} \sigma}{\mathrm{P}^{2} \mathrm{~d} \Omega \frac{\mathrm{dP}}{\mathrm{E}}}=\frac{\sigma_{\mathrm{O}}}{\Delta^{2}} \mathrm{~F}(\mathrm{x}) \mathrm{G}\left(\mathrm{P}_{\mathrm{T}}\right)
$$

Let

$$
\begin{aligned}
\mathrm{N}_{\mathrm{i}}= & \text { number of incident particles } \\
\mathrm{N}_{\mathrm{d}}= & \text { number of detected outgoing particles } \\
\mathrm{F}_{\mathrm{i}}= & \text { probability that an incident particle interacts } \\
\mathrm{F}_{\mathrm{d}}= & \text { probability that, given an interaction, } \\
& \text { a secondary particle will enter our detector. }
\end{aligned}
$$

Then we have

$$
\begin{aligned}
& \mathrm{N}_{\mathrm{d}}=\mathrm{F}_{i} \mathrm{~F}_{\mathrm{d} \mathrm{N}_{i},} \\
& \mathrm{~F}_{i}=5 \% \text { for } \sigma_{0}=30 \mathrm{mb} \text { with a } 30 \mathrm{~cm} \text { 1iquid hydrogen target, }
\end{aligned}
$$

and

$$
F_{d}=F(x) G\left(P_{T}\right) \frac{P^{2} d \Omega}{\Delta^{2}} \frac{d P}{E}
$$

Thus we see that the quantity $\frac{\mathrm{P}^{2} \mathrm{~d} \Omega}{\Delta^{2}} \frac{\mathrm{dP}}{\mathrm{E}}$ measures the acceptance of our apparatus, over the entire region of laboratory angles and momenta. Assuming that our apparatus is only able to handle a single particle at a time, we want to make $\frac{\mathrm{P}^{2} \mathrm{~d} \Omega}{\Delta^{2}} \frac{\mathrm{dP}}{\mathrm{E}}$ sma11 compared to unity in order to ensure that there is only a smal1 probabiltiy of detecting two particles from a single event. The best we can do is to reject any event for which there are two or more particles seen, and apply an appropriate correction to the overall normalization. If there are no correlations we do not thereby make any error, but since in general there may be correlations between 
particles in various regions of phase space, we want to be sure that the fraction of events thus rejected is small.

As for the sensitivity to small cross-sections, the apparatus to be described in this proposal will be characterized by an acceptance of

$$
\frac{\mathrm{P}^{2} \mathrm{~d} \Omega}{\Delta^{2}} \frac{\mathrm{dP}}{\mathrm{E}} \approx 1 \times 10^{-2}
$$

roughly independent of momentum. We take the lower limit of our experimental sensitivity to be given by a cross-section such that we detect 100 events per hour, or about 0.1 event per pulse at NAL. Assuming a $30 \mathrm{mb}$, tota 1 cross-section and a $30 \mathrm{~cm}$. 1iquid hydrogen target, we thus have an experimental sensitivity extending down to

$$
\left.F(x) G\left(P_{T}\right)\right]_{\min }=2 \times 10^{-6} \times\left[\frac{10^{8} \text { particles } / \text { pulse }}{\text { incident Beam Rate }}\right]
$$

or, in terms of the minimum detectable laboratory cross-section,

$$
\left.\frac{\mathrm{d} \sigma}{\mathrm{d} \Omega \mathrm{dP}}\right]_{\min }=3.7 \times 10^{-29} \frac{\mathrm{cm}^{2}}{\mathrm{Sr}-\mathrm{Gev} / \mathrm{c}} \times \frac{10^{8} \text { particles } / \text { pulse }}{\text { Incident Beam Rate }} \times \frac{\mathrm{P}}{100 \mathrm{Gev} / \mathrm{C}}
$$

It should be remembered that, although pion and proton rates will

indeed be $10^{8} /$ pulse or higher, the rates of $\bar{p}^{\prime} s$ and $K^{\prime}$ 's will be considerably less, and thus we will need all the experimental sensitivity we can get to be able to obtain useful information pith these relatively rare particïs. 
6. BACKGROUNDS AND MAXIMUM BEAM INTENSITY

There will be a maximum beam intensity above which accidental rates will be excessive. The principal limitation is that singles rate in the wire chambers. Using multi-wire proportional chambers with $\sim 100 \mathrm{nsec}$ time resolution, singles rates of $\sim 10^{6}$ counts/second can be handled. A typical event will then contain the tracks of a true secondary particle within our momentum acceptance, along with accidental counts from particles that registered in a few of the chambers only. Our events are considerably over-constrained, so we can easily reject a few spurious counts. Specifically, we require that the input track form a straight line pointing to the target, the output track from a straight line, and that the two tracks meet properly inside the magnet gap.

The background may be divided into two categories: secondary particles produced in our target, and background not associated with our target.

Using the rate estimate developed in Section 5 , we can set an upper limit on the singles rate from secondary particles. Consider a beam of $\mathrm{N}_{i}$ particles per second incident on a $30 \mathrm{~cm}$. 1iquid hydrogen target, with a small counter of area $a^{2}$ located a distance $\mathrm{L}$ downstream and $\mathrm{b} \ll \mathrm{L}$ transverse to the beam (see Figure 4). The singles rate will then be, according to the model of Section 5, 


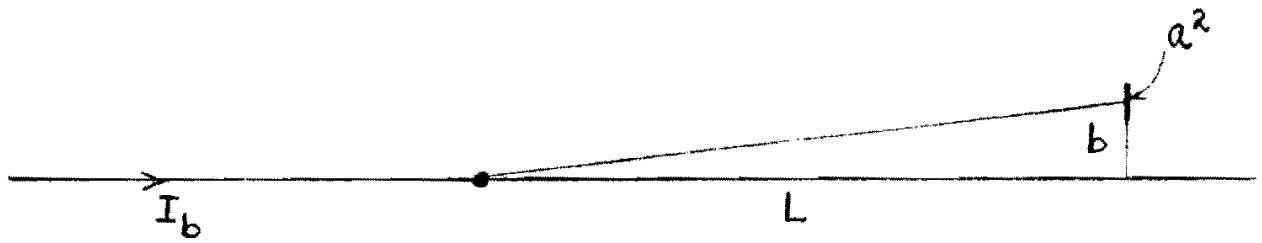

Figure 4. Illustrating the singles rate calculation of section 6 . 


$$
\mathrm{N}_{\mathrm{s}}=\mathrm{F}_{i} \mathrm{~F}_{\mathrm{d}} \mathrm{N}_{\mathrm{i}}
$$

where $\mathrm{F}_{\mathrm{i}}$ will be $\sim 5 \%, \mathrm{~F}_{\mathrm{d}}$ is given by

$$
F_{d}=\int_{0}^{P} F(x) G\left(P_{T}\right) \frac{P^{2} a^{2}}{\Delta^{2} L^{2}} \frac{d P}{E}
$$

and

$$
\mathrm{P}_{\mathrm{T}}=\frac{\mathrm{b}}{\mathrm{L}} \mathrm{P}
$$

To obtain an upper limit on $F_{d}$, we replace $F(x)$ by $1, E$ by $P$ and we extend the upper limit of integration to infinity. Then we find, using the normalization condition on $G$ from section 5 ,

$$
F_{d} \leq \int_{0}^{\infty} G\left(\frac{b}{L} P\right) \frac{P^{2} a^{2}}{\Delta^{2} L^{2}} \frac{d P}{P}=\frac{a^{2}}{b^{2}} \int_{0}^{\infty} \frac{u G(u) d u}{\Delta^{2}}=\frac{a^{2}}{2 \pi b^{2}}
$$

This upper limit on the singles rate is independent of the longitudinal distance $L$, thus encouraging us to use a variable length spectrometer.

Our design is such that, with the apparatus set at the minimum accessible value of $\mathrm{P}_{\mathrm{T}}$,

$$
\frac{\mathrm{a}^{2}}{2 \pi \mathrm{b}^{2}}=0.07
$$

(see Section 8 ). Thus we find that target-associated background limits the beam rate to $0.5 \times 10^{8}$ particles per second at the lowest $\mathrm{P}_{\mathrm{T}}$ setting. 
It is difficult to give a precise estimate of the effects of background from beam halo and general room background. The maximum useable beam intensity will in any case depend on the momentum setting and angle of the spectrometer. On the basis of our experience at lower energy accelerators, we feel that we can safely expect the spectrometer to be able to handle an incident beam intensity of $10^{8}$ particles/second. 
7. PARTICLE IDENTIFICATION

We plan to use threshold Čerenkov counters to identify protons and kaons. Such a counter is simply a length of tubing, filled with gas at the appropriate pressure, with a $45^{\circ}$ mirror and a phototube at the downstream end. Different lengths will be used depending on the momentum range being studied, as discussed below. Wire chambers before and after the counters will ensure that only one particle has traversed the counter, and will help reject spurious counts arising from interactions with the gas and windows. At the very lowest momenta we may also use dE/dx or time of flight to identify particles. Rejection ratios of $10^{4}: 1$ should be obtained readily.

Muons and electrons will be identified by means of a shower counter for electrons followed by a thick absorber and a counter for muons. Quark identification is discussed in Section 9.

Threshold Cerenkov counters were built by Gorin et a1 $1^{10}$ for use at Serpukhov, using quartz optics. Their measured threshold curves correspond to an average number of photoelectrons of $\mathrm{N}_{\mathrm{e}}=1.6 \times 10^{4} \cdot{ }^{2} \mathrm{~L}$

where $\theta$ is the Cerenkov angle and $L$ is the counter length in meters. Useful signals were obtained from single photoelectrons. We assume that we can equal this performance.

To identify pions at momenta below some maximum value $P_{\max }$, there will be a counter whose threshold for counting $K^{\prime} s$ is at $\mathrm{P}_{\max } \cdot$ If we demand an average of 10 photoelectrons from pions 
of momentum $\mathrm{P}_{\max }$, we find that the required length is

$$
L=29\left(\frac{P_{\max }}{100}\right)^{2} \text { meters }
$$

where $\mathrm{P}_{\max }$ is in Gev/c. The $\breve{C}$ erenkov angle is

$$
A=4.7 \times 10^{-3}\left(\frac{100}{P_{\max }}\right) \text { radians }
$$

and the radius of the Cerenkov cone is

$$
a={ }_{A} L=0.14\left(\frac{\mathrm{P}}{100}\right) \text { meters } .
$$

The counter will cease counting pions entirely at a momentum of

$\frac{\mathrm{m}_{\pi}}{\mathrm{m}_{\mathrm{K}}} \mathrm{P}_{\max }=0.28 \mathrm{P}_{\max } ;$ it will give 7 photoelectrons $\left(\mathrm{e}^{-7}=0.9 \times 10^{-3}\right)$ at a momentum of

$$
\mathrm{P}_{\min }=0.51 \mathrm{P}_{\max }
$$

A second such counter, set at proton threshold, will identify kaons. We find

$$
\begin{aligned}
& L=10\left(\frac{P_{\max }}{100}\right)^{2} \\
& \theta=8.0 \times 10^{-3}\left(\frac{100}{P_{\max }}\right) \\
& a=0.08\left(\frac{P_{\max }}{100}\right)
\end{aligned}
$$

and

$$
\mathrm{P}_{\min }=0.72 \mathrm{P}_{\max }
$$


34

To find the amount of gas required we use

$$
\begin{aligned}
\mathrm{n}-1 & =\frac{\mathrm{m}^{2}}{2 \mathrm{P}_{\max }^{2}} \\
& =\left\{\begin{array}{l}
1.2 \times 10^{-5}\left(\frac{100}{\mathrm{P}_{\max }}\right)^{2} \text { for separating } \mathrm{k}^{\prime} \mathrm{s} \text { from } \pi^{\prime} \mathrm{s} \\
4.5 \times 10^{-5}\left(\frac{100}{\mathrm{P}_{\max }}\right)^{2} \text { for separating } \mathrm{p}^{\prime} \mathrm{s} \text { from } \mathrm{k}^{\prime} \mathrm{s}
\end{array}\right.
\end{aligned}
$$

Since the gas pressure goes as $1 / P_{\max }{ }^{2}$ while the counter length goes as $\mathrm{P}_{\max }{ }^{2}$, the number of grams per $\mathrm{cm}^{2}$ is independent of $\mathrm{P}_{\max }$. For example for air or $\mathrm{CO}_{2}$ we require $0.1 \mathrm{~g} / \mathrm{cm}^{2}$ of Čerenkov radiator for each of the two counters. 
8. SYSTEM ACCEPTANCE, RESOLUTION AND OTHER PERFORMANCE FACTORS.

Figure 5 is a simplified view of the spectrometer, with longitudinal and transverse distance parameters $L, L^{\prime}, a$, and $b$ defined. Let $P_{0}$ denote the central momentum (the momentum of a particle which goes through the center of each chamber). The solid angle acceptance at $P_{0}$ is then determined by the aperture of the last chamber, (a square of side a), and has the value

$$
\mathrm{d} \Omega_{\mathrm{O}}=\frac{\mathrm{a}^{2}}{\mathrm{~L}^{2}}
$$

The overall scale of the system is determined by the quantity $\mathrm{L} / \mathrm{P}_{\mathrm{O}}$, which in our design has the value

$$
\frac{\mathrm{L}}{\mathrm{P}_{\mathrm{O}}}=1.87 \text { meters } / \mathrm{Gev}
$$

Since we vary the spectrometer linearly with momentum, the transverse momentum acceptance $\mathrm{P}_{0}^{2} \mathrm{~d}_{\Omega_{0}}$ is independent of $\mathrm{P}_{0}$. The solid angle acceptance as a function of momentum is shown in Figure 6 . There is a $2: 1$ range of useful acceptance extending from

$$
\mathrm{P}_{\min }=0.75 \mathrm{P}_{\mathrm{o}}
$$

to

$$
\mathrm{P}_{\max }=1.5 \mathrm{P}_{\mathrm{O}} \text {. }
$$

The solid angle averaged over this range is $\overline{\mathrm{d} \Omega}=0.84 \mathrm{~d} \Omega_{0}$, and so the acceptance in invariant phase space is, for $a=0.1$ meter, 


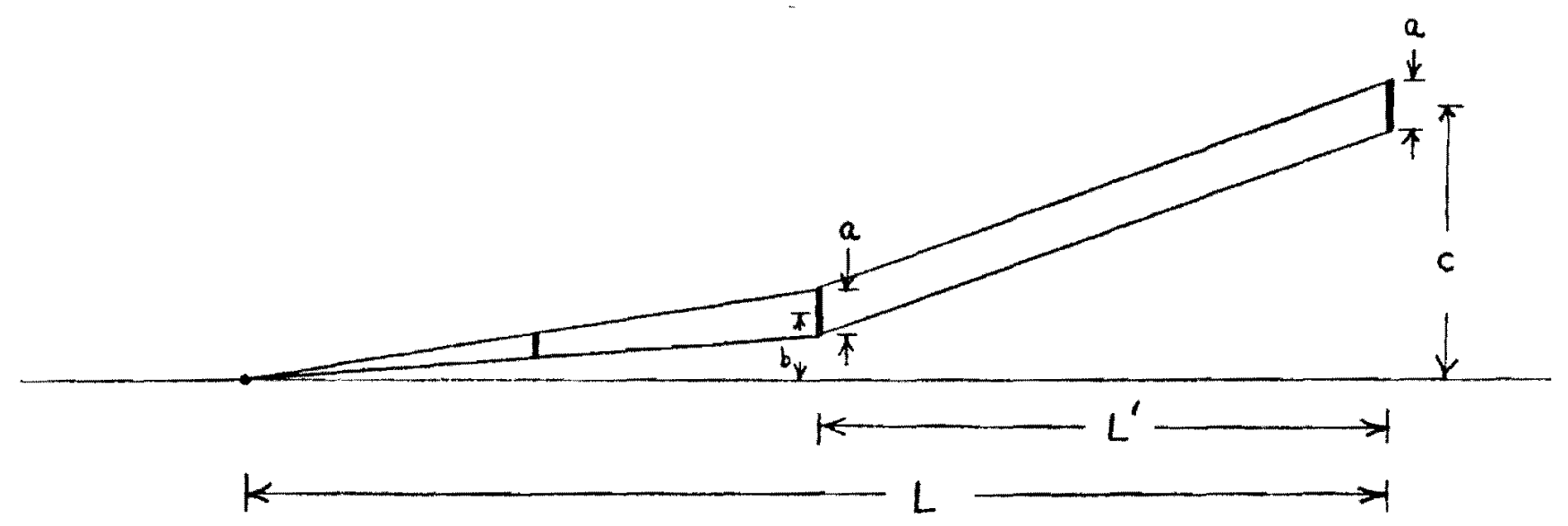

Figure 5. Simplified view of the spectrometer

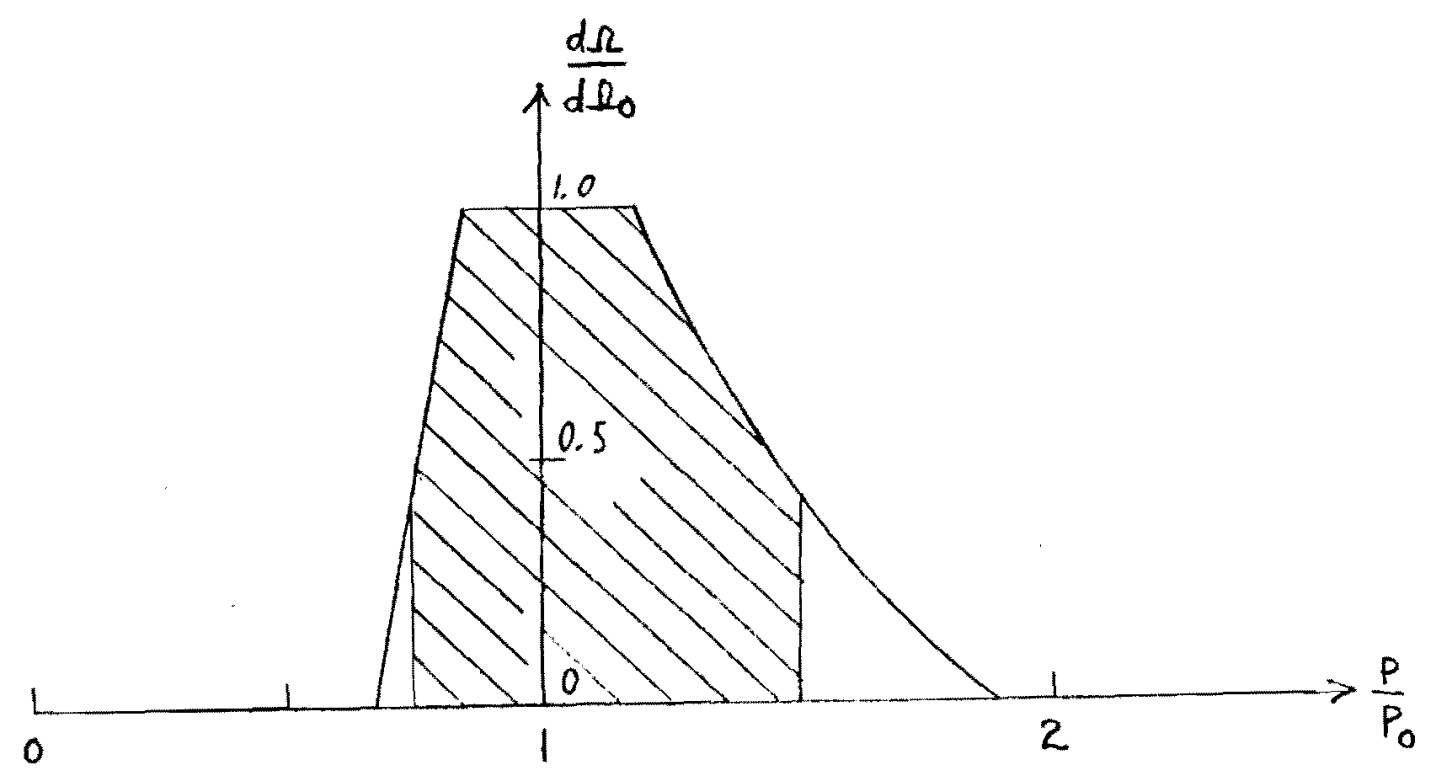

Figure 6. Solid angle versus momentum. The region from $\mathrm{P} / \mathrm{P}_{\mathrm{O}}=0.75$ to $\mathrm{P} / \mathrm{P}_{\mathrm{o}}=1.5$ is shaded. 


$$
\begin{aligned}
\mathrm{P}^{2} \mathrm{~d}\left(\frac{\mathrm{dP}}{\mathrm{E}}\right. & =\mathrm{P}_{\mathrm{o}}^{2} \times 0.84 \overline{\mathrm{d} \Omega} \frac{1.5 \mathrm{P}_{\mathrm{o}}-0.75 \mathrm{P}_{\mathrm{O}}}{\mathrm{P}_{\mathrm{o}}} \\
& =1.8 \times 10^{-3}(\mathrm{Gev} / \mathrm{c})^{2}
\end{aligned}
$$

independent of $\mathrm{P}_{\mathrm{O}}$. Expressed in terms of the characteristic transverse momentum $\Delta=0.4 \mathrm{Gev} / \mathrm{c}$ of section $t$, the acceptance is

$$
\frac{\mathrm{P}^{2} \mathrm{~d} \Omega}{\Delta^{2}} \frac{\mathrm{dP}}{\mathrm{E}}=1.1 \times 10^{-2}
$$

The minimum detectable transverse momentum at $\mathrm{P}=\mathrm{P}_{\mathrm{O}}$ depends on the transverse distance $b$ in Figure 5. For the value $\mathrm{b}=0.15$ meters it has the value

$$
\mathrm{P}_{\mathrm{T}, \min }=0.13 \mathrm{Gev} / \mathrm{c}
$$

The quantity used in Section 6 to put an upper limit on the target-associated singles rate is

$$
\frac{\mathrm{a}^{2}}{2 \pi \mathrm{b}^{2}}=0.07
$$

The magnet $\mathrm{M}_{1}$ has a transverse momentum impulse of

$$
\Delta \mathrm{P}_{\mathrm{mag}}=0.32 \mathrm{Gev} / \mathrm{c}=0.7 \text { meters } \times 15 \mathrm{Kg}
$$

The momentum resolution is then determined by two factors: wire chamber resolution and multiple scattering. In a simple worst-case model, we assume that only the information from the 
end chambers $W_{1}, W_{3}, W_{4}$, and $W_{6}$ is used, and that the momentum is determined from the difference of the slopes before and after the magnet. Assuming an r.m.s. position resolution of $0.5 \mathrm{~mm}$ in each chamber, the momentum resolution due to chamber position resolution is

$$
\left.\frac{\delta \mathrm{P}}{\mathrm{P}}\right]_{\text {chamber }}=0.5 \% \text {, }
$$

independent of $\mathrm{P}_{0}$. The momentum resolution due to multiple scattering depends on the quantity $\Delta \mathrm{P}_{\text {scatt }}$, the r.m.s. transverse momentum from multiple scattering, through the relationship

$$
\left.\frac{\delta \mathrm{P}}{\mathrm{P}}\right]_{\text {scatt }}=\frac{\Delta \mathrm{P}_{\text {scatt }}}{\Delta \mathrm{P}_{\text {mag }}}
$$

The quantity $\Delta \mathrm{P}_{\text {scatt }}$ is the sum of contributions added in quadrature from multiple scattering in the chambers, Čerenkov counter and vacuum pipe windows, Čerenkov counter gas, and air gaps along the entire path from the first to the last chamber. The scattering contribution from each element is weighted by a factor which varies linearly from unity for components near the magnet to zero for components near $W_{1}$ or $W_{6}$. We calculate

$$
\Delta \mathrm{P}_{\text {scatt }}=0.02 \mathrm{Gev} / \mathrm{c} ;\left.\frac{8 \mathrm{P}}{\mathrm{P}}\right|_{\text {scatt }}=0.6 \%
$$

and the overall momentum resolution is

$$
\frac{\delta P}{P}=0.8 \%
$$


This value is more than adequate for the physics we are studying. The multiple scattering quoted assumes that we can make plane, $45^{\circ}$ mirrors for our Cerenkov counters by depositing a suitable reflective surface on thin foils. We have not yet verified this assumption by actually making such a mirror; however even if a thin glass mirror were required, $\frac{\delta \mathrm{P}}{\mathrm{P}}$ would be acceptable.

The momentum bite is well matched to the useful momentum range of the pion Cerenkov counters. We will need two kaon Cerenkov counters, set at different pressures, in order to cover the entire momentum range for kaons at once.

The distance $L^{\prime}$ must be enough to accomodate the Cerenkov counters. This condition can be met up to some maximum momentum. If there is to be enough room to accomodate the pion counter and one kaon counter (the other kaon counter can, at the highest momenta, be put just before the bending magnet or just after the last chamber), we must have

$$
(29+10)\left(\frac{\mathrm{P}_{\max }}{100}\right)^{2} \leq \mathrm{L}^{\prime}=\frac{1}{2} \times 1.87 \mathrm{P}_{\mathrm{o}}=.62 \mathrm{P}_{\max } \cdot
$$

Thus

$$
\mathrm{P}_{\max }=160 \mathrm{Gev} / \mathrm{c}
$$

is the highest momentum at which we can have $10^{4}: 1$ particle identification over the full momentum acceptance of the 
spectrometer. Identification can be accomplished at higher momenta if separation only up to momentum $P_{0}$ is required:

$$
\begin{aligned}
(29+10)\left(\frac{P_{o}}{100}\right)^{2} & \leq L^{\prime}=\frac{1}{2} \times 1.87 \mathrm{P}_{\circ} \\
P_{O} & \leq 250 \mathrm{Gev} / \mathrm{c}
\end{aligned}
$$

We can reach $300 \mathrm{Gev} / \mathrm{c}$ by relaxing our requirements to $10^{3}: 1$ identification. 


\section{1,42}

9. BEAM SURVEY AND QUARK SEARCH

Our principal interest, and the objective around which our apparatus has been designed, is the study of inelastic scattering phenomena with a proton target and a variety of incident particles. Our apparatus can also play a useful role in the intital program of beam surveys and searches for new particles which may be carried on before secondary beams become available.

We believe that it is logical to divide such a program of beam surveys into several complementary experiments. Our apparatus is well adapted to a broad survey of the high-rate, low momentum transfer region. It will quickly provide the engineering information needed for beam design, and it wil1 detect quarks if they are produced $\geq 10^{-4}$ times as copiously as $\overline{\mathrm{P}}$ 's.

The apparatus will be set up in its $100-200 \mathrm{Gev} / \mathrm{c}$ configuration (250 meters overall length), with 200 Gev protons from the external proton beam incident on a nuclear target. Coverage will be obtained down to $25 \mathrm{Gev} / \mathrm{c}$ secondary momentum in three momentum bands obtained with full, one half, and one fourth of the design magnetic field. The momentum resolution and acceptance for lower bands will be less than in the variable length design, but will still be more than adequate for a beam survey. 
Data will be taken at constant lab angle. Coverage of the desired angular range will be obtained by means of transverse motion of the spectrometer components as discussed in the previous section. The required floor width of the experimental area is of course proportional to the maximum 1aboratory angle we wish to reach. To obtain coverage out to $15 \mathrm{mrad}$, we need an area extending out 3.5 meters on one side of the beam center line.

If the needed transverse room is not available, we can keep the spectrometer components fixed over the beam line and use deflecting magnets to vary the transverse momentum, as discussed in Section 3.

The apparatus can easily seek quarks simultaneously with the beam survey. Assuming a minimum detectable quark rate of 1 per hour $\left(\left.\frac{\mathrm{d} \sigma}{\mathrm{d} \Omega \mathrm{dp}}\right|_{1 \mathrm{ab}} \approx 10^{-31} \mathrm{~cm}^{2} /\right.$ sterad-Gev/c), rejection ratios of roughly $10^{8}: 1$ against $\pi^{-}, \mathrm{K}^{-}, \overline{\mathrm{P}}$, and antideuterons will be required.•

Anticoincidence with the Cerenkov counters for identifying $\pi, K$ and $\bar{p}$ should provide at least $10^{4}: 1$ rejection. Two $d E / d x$ counters set to accept particles with ionization rates of .1 to .6 minimum will reject the leakage of $\pi^{-}, \mathrm{K}^{-}$and $\overline{\mathrm{P}}$ from the Cerenkov counters, and also any antideuterons, antiheliums, etc. Assuming 225 photoejectrons and $10 \%$ uniformity of pulse height across the counters, a rejection ratio of $10^{4}: 1$ for 
each counter is calculated. Because of the Landau tail the efficiency for detecting charge $2 / 3$ quarks will be about 50 per cent. (These counters can also be gated to accept particles of charge 2 or greater, and thus count antihelium. The wire chambers would verify that only one particle was being counted.)

A third $d E / d x$ counter will be pulse height analysed with each event. The resulting spectrum for events satisfying the quark trigger would show peaks with a characteristic Landau distribution from charge $1 / 3$ and $2 / 3$ quarks, as well as any particles leaking through the trigger.

The momentum of any quarks found will be known from their trajectories through the spectrometer. A rough value for the quark mass will be attained by measuring the particle's time of flight through the apparatus. For example, a charge $2 / 3$ quark with $50 \mathrm{Gev} / \mathrm{c}$ true momentum will have a time of flight difference of $1.5\left[\mathrm{M}_{\text {quark }} / 5 \mathrm{Gev} / \mathrm{c}^{2}\right]^{2} \mathrm{nsec}$. Of course, if a tantalizing peak is observed, the precise mass will be determined by a Cerenkov counter pressure curve.

The maximum quark mass observable in the reaction $p p \rightarrow p p q \bar{q}$ at $200 \mathrm{Gev} / \mathrm{c}$ beam momentum is

$$
\mathrm{M}_{\text {quark }}=.5\left(\sqrt{\mathrm{s}}-2 \mathrm{M}_{\mathrm{P}}\right) \approx 8.8 \mathrm{Gev} / \mathrm{c}^{2} .
$$


This mass quark would have a lab momentum of

$$
\beta_{\mathrm{cm}} \gamma_{\mathrm{cm}} M_{\text {quark }} \approx 90 \mathrm{Gev} / \mathrm{c} .
$$

For charge $2 / 3$ and $1 / 3$ quarks this corresponds to spectrometer momentum settings of 135 and $270 \mathrm{Gev} / \mathrm{c}$, respectively. Lower mass quarks would have a range of lab momenta. For example, a $5 \mathrm{Gev} / \mathrm{c}^{2}$ quark could have lab momenta between 16 and $165 \mathrm{Gev} / \mathrm{c}$. 
10. RUNNING TIME

A. Beam Survey - 150 hours tota1

Setup and counter tests -100 hours

Survey and quark search - 50 hours

Laboratory angles of $2.5,3.5,7.5$ and $15 \mathrm{mr}$ will

be covered for secondary momenta of 25-50,50-100, and 100-200 Gev/c. Crude rate estimates indicate that we will get better than $10 \%$ statistics on particle yields for any feasible NAL beam in the momentum range 25-200 Gev/c. The quark search will be combined with the negative particle survey at 2.5 and $3.5 \mathrm{mr}$ for the highest momentum range, and will take 20 hours.

\section{B. Forward Spectrometer-total 450 hours}

Testing - 30 hours (in addition to the beam survey setup time).

Changeover checkout and counter efficiency tests70 hours ( 7 configurations, and 12 hours for each changeover). Running time - $350 \mathrm{hrs}$.

We plan to cover the range of secondary momenta from 1.25 to $160 \mathrm{Gev} / \mathrm{c}$ in 7 configurations. For each configuration, beam momenta of 160,80 and $40 \mathrm{Gev} / \mathrm{c}$ will be used (except, of course the high momentum configurations won't be used at lower beam momenta). Cross-sections for six to eight transverse 
momenta, and four charge combinations (beam + and -, secondary + and -) will be measured. Individual runs will take from 20 min. to $1 \frac{1}{2}$ hours. We expect to keep empty target rates to less than $10 \%$ of full target rates using our ability to trace rays through the apparatus. A modest allotment of empty target time is included in the running time estimate.

C. Backward Spectrometer - tota1 250 hours

Setup - 50 hours

Running time - 200 hours

Measurements will be taken at 8 angles between $25^{\circ}$ and $155^{\circ}$, two secondary momentum ranges, 4 charge combinations and 3 beam momenta.

Tota1 time requested - 850 hours. 


\section{BEAM REQUIREMENTS}

In this section we discuss beam requirements for the study of inelastic hadron-hadron scattering assuming that the accelerator operates at $200 \mathrm{Gev} / \mathrm{c}$. Our spectrometer is capable of studying inelastic scattering at secondary momenta up to $500 \mathrm{Gev} / \mathrm{c}$ with particle identification up to $250 \mathrm{Gev} / \mathrm{c}$. We are prepared to contribute to the beam survey program and to study inelastic scattering with $500 \mathrm{Gev} / \mathrm{c}$ protons if they are available.

We plan to study inelastic scattering at incident momenta of 40,80 , and $160 \mathrm{Gev} / \mathrm{c}$. We require an unseparated beam of positive or negative particles with momentum spread $\frac{\Delta p}{P}= \pm .005$. The beam must have a drift space where the divergence is $1 \in s s$ than \pm .02 mrad to accomodate a DISC Cerenkov counter which can distinguish $\pi^{\prime} \mathrm{s}$ and $\mathrm{K}^{\prime} \mathrm{s}$ at $160 \mathrm{Gev} / \mathrm{c}$.

The permissable beam divergence at the final image is determined by the uncertainty in the transverse momentum of the incident particle. The divergence must therefore be the smallest at the highest beam momentum and most forward spectrometer geometry. If we limit the uncertainty in transverse momentum under these conditions to $\pm 50 \mathrm{Mev} / \mathrm{c}$ we obtain a divergence less than or equal to \pm 0.25 mrad. This divergence can be achieved by turning off the focusing magnets between the DISC counter and the hydrogen target and using a large (several centimeter) diameter target. At lower beam momentum or lower secondary laboratory 
momentum the divergence at the final image can be larger. When the backward spectrometer is used the hydrogen target should be smaller in diameter (one centimeter) in order that the lowest momentum secondaries see the least possible material in getting out of the target.

The maximum intensity of the beam is 1 imited by the background, and by the maximum rate at which beam particles can be identified. As discussed in Sec. 6 , the experiment can use a beam of $10^{8}$ particles/pulse or more.

A beam similar to the $2.5 \mathrm{mrad}$ beam described by Reeder and MacLachlan in the 1969 NAL Summer Study is adequate for our experiment.

For the beam survey, the properties of the incident proton beam must be consistent with the above requirements. In particular, we must be able to reduce the incident proton intensity to $10^{8}$ or $10^{9}$ protons/pulse. 
12. EQUIPMENT REQUIREMENTS AND MANPOWER

1) Experimenta1 Area: The experimental area should be 4 to 5 meters wide to one side of the incident beam and 200 meters long. It should include a portable house or trailer furnished by NAL for housing electronics.

2) Liquid Hydrogen Target: We will require a liquid hydrogen target with appendices of two different diameters for the various beam divergences and magnifications used. There must be two appendices of each size of which one is filled with hydrogen and one is empty for target empty runs. We must be able to switch targets by remote control. The targets should be provided by NAL.

3) Spectrometer Magnet: The spectrometer magnet is a C-magnet of a type presently in use at the Lawrence Radiation Laboratory. We expect NAL to borrow an existing magnet or construct a similar one.

4) Spectrometer Mounting and Alignment System: A1though this system is simple in principle, it will require a larger engineering effort than we can mount ourselves. For the "forward" spectrometer the motion and alignment of the magnet and detectors transverse to the beam must be accomplished by remote control. Motion parallel to the beam should also be accomplished easily, but will be performed the minimum number of times because a change in 
length of the Cerenkov counters is made at these times. The "backward" spectrometer is simpler in that it pivots about the hydrogen target. It is desirable to control the motion of the backward spectrometer remotely.

We request that NAL provide the spectrometer mounting and alignment system.

5) DISC Cerenkov Counter: The DISC Cerenkov counter for the beam will be provided by the experimenters.

6) Computer Facilities: We require a small computer for data collection and monitoring. We are prepared to furnish this computer ourselves. We will also require fast turn-around (a few hours) access to a large computer for data processing. In addition to the three authors of this proposal we will have one additional $\mathrm{Ph}$. D. physicist (a research associate), one student, and one technician. 


\section{BIBLIOGRAPHY}

1. R.P. Feynman, in the Proceedings of the Third Internationa1 Conference on High Energy Collisions at Stony Brook, and Phys. Rev. Letters 23, 1415, (1969).

2. E. Fermi, Progress of Theoretical Physics $\underline{5}, 570$ (1950).

3. J. Benecke, T.T. Chou, C.N. Yang, and E. Yen, Phys. Rev. 188, 2159 (1969).

4. See a1so the talks by J.D. Bjorken and S. Dre11, Proceedings of the International Conference on Expectations for Particle Reactions at the New Accelerators, April 1970.

5. See F. Gilman, Proceedings of the 4th International Symposium on Electron and Photon Interactions at High Energy, Daresbury Nuclear Physics Laboratory, Daresbury nr. Warrington, England (1969), Ed. D. Braben, and references therein.

6. Diddens et a.1, Nuovo Cimento 31,961 (1964); Dekkers et a1, Phys. Rev. 137, B962 (1965); Anderson et a1, Phys Rev. Letters 19, 198 (1967); Ratner et a1, Phys. Rev. 166, 1353 (1968); A1laby et a1, CERN PS/6517/k1 (submitted to the 14th Conference on High Energy Physics, Vienna, 1968); Day et a1, Phys. Rev. Letters 23, 1055 (1969).

7. A.R. Erwin, Proceedings of the Internationa1 Conference on Expectations for Particle Reactions at the New Accelerators, April 1970 . 
8. R. Hagedorn and J. Ranft, Supp1. Nuovo Cimento $\underline{6}, 169$ (1968)

9. D. Amati, A. Stanghellini and S. Fubini, Nuovo Cimento XXVI, 896 (1962); G. F. Chew and A. . Pignotti, Phys. Rev. 176, 2112 (1968); S. Pinsky and W. I. Weisberger (unpub1ished).

10. Yu P. Gorin, S. P. Denisov, S. V. Donskov, A. F. Juanaitsev, A. I. Petrukhin, Yu. P. Prokoshkin, D. A. Stoyanova and R. S. Shuvalov, IHEP Preprint 69-63 (English translation Scientific Translation Service, Order No. 8624, Ann Arbor, Michigan). 
Eugene W. Beier, David L. Kreinick and Howard Weisberg

\section{A Guide for the Reader}

The essence of our proposal is contained in its first three sections, which may be read as a self-contained unit. The remaining nine sections contain supporting information.

\section{Points to be Emphasized}

The physics is exciting. According to ideas of Yang on Limiting Fragmentation and of Feynman on Partons, hadron-hadron scattering should approach a limiting behavior at NAL energies. Further simple kinds of behavior should also be found, especially target independence and $1 / x$ longitudinal momentum dependence (which is intimately related to phenomena observed in inelastic electron scattering). The elucidation of this behavior could be one of the most exciting early results in hadron physics from N.A.L.

Our experimental design is simple and straightforward and our requirements modest (the overall length is discussed below). We are well aware that the history of this type of experiment has been marked by a number of incorrect measurements and we feel confldent in our ability to avoid systematic errors, while carrying out the experiment in the allotted time.

\section{The Overall Length}

Our design length of 200 meters was arrived at by requiring $\pi-k$ separation at $160 \mathrm{Gev} / \mathrm{c}$ with 10 photoelectrons in a threshold Cerenkov counter, assuming the number of photoelectrons from a counter of length L cm is $N_{e}=160 \theta^{2} L$. Several N.A.L. proposals are more optimistic and hence come up with shorter design lengths, while on the other hand a conversation with G. Meunier suggests that even $160 \theta^{2} \mathrm{~L}$ may be difficult. In any case, we can give up $\pi-K$ separation at large $x$ at the highest beam momentum, and thus make our overall length the same as that of other proposed 160 Gev/c spectrometers, without seriously compromising our physics objectives. 
Q

IV. A Comparison of Proposed NAL Experiments on Particle Production Spectra.

\begin{tabular}{|c|c|c|c|c|c|}
\hline EXPERIMENT & PROJECTILES & $\begin{array}{l}\text { DETECTED } \\
\text { PARTI CLES }\end{array}$ & $\begin{array}{l}\text { COVERAGE } \\
\text { IN } X\end{array}$ & $\begin{array}{l}\text { MAXIMUM } \\
P_{T}, \text { Gev/c }\end{array}$ & $\begin{array}{l}\text { CAN GET PARTICLE } \\
\text { MULTIPLICITY? }\end{array}$ \\
\hline 14 - Franzini & $p$ & $\mathbf{p}$ & $-1<x<-0.7$ & 0.34 & no \\
\hline 23 - Rothberg & $\pi$ & $\pi^{ \pm}, k^{ \pm}$ & $0<x<0.4$ & 0.5 & no \\
\hline 52 - Weisberg & $\pi^{ \pm}, k^{t}, p^{ \pm}$ & $\pi^{ \pm}, K^{ \pm}, p^{t}$ & $-1<x<1$ & $\begin{array}{l}1-3.5 \\
\text { (depends on } x)\end{array}$ & yes \\
\hline 63 - Walker & $p$ & $\begin{array}{l}\pi_{ \pm}^{ \pm}, k_{+}^{ \pm}, p^{ \pm}, \\
\mu^{ \pm},\end{array}$ & $\begin{array}{l}-1<x<-0.2(p) \\
-1<x<-0.05(k) \\
-1<x<+0.01(\pi)\end{array}$ & 2.4 & $\begin{array}{l}\text { no } \\
\text { no } \\
\text { yes }\end{array}$ \\
\hline 64 - Read & p & $x^{ \pm}, k^{ \pm}, p^{ \pm}$ & $0.1<x<1$ & 4 & no \\
\hline
\end{tabular}


ADDENDUM TO N.A.L. PROPOSAL 52

Eugene W. Beier, David L. Kreinick, Howard Weisberg University of Pennsylvania

November 16,1970

We have proposed an experiment to measure the differential crosssection for single particle production in the collisions of charged particles with protons. Specifically we proposed to measure $\frac{\mathrm{d} \sigma}{\mathrm{d}^{3} \mathrm{p}_{\mathrm{c}}}$ for the reaction $a+p \rightarrow c+$ anything, where $a, c=p^{ \pm}, k^{ \pm}$and $\pi^{ \pm}$, over the complete range of secondary momenta. The theoretical considerations which motivated our proposal strongly suggest that there is great interest in covering the widest possible range of secondary momenta. It was suggested by Dr. Wilson that we consider the use of the single-arm spectrometer facility, or the forward arm from the doublearm spectrometers of experiments 7 and 61 , to carry out our measurements. As discussed further below, these spectrometers are essentially high-momentum devices, and will be mainly limited to studying particles moving with large positive momentum in the $\mathrm{c} . \mathrm{m} .(\mathrm{x}>0$, or "Fragments of the Projectile" in Yang's language of Limiting Fragmentation). We wish to emphasize here the importance, among early measurements from N.A.L., of measuring the momentum spectra of particles having relatively sma11 laboratory momenta (and correspondingly large angles). Specifically we refer to particles having momenta near zero in the c.m. (the "wee" momenta which according to Feynman play a fundamental role in hadronhadron scattering, and particles of negative x ("Fragments of the Target"). 
It is to be emphasized that there is separate physical interest in measurements in each of the kinematic regions, and that measurements taken in different regions will complement each other. We believe that early measurements from N.A.L. should not be 1 imited to the high-momentum sma11 angle region only.

We suggest that the kinematic region be covered in three phases:

I. Particles going forward in the $1 \mathrm{ab}$ with $1.5 \leq \mathrm{p}_{1 \mathrm{ab}} \lesssim 20 \mathrm{Gev} / \mathrm{c}$.

II. Low momentum, large angle particles $\left(\mathrm{p}_{1 \mathrm{ab}} \lesssim 1.5 \mathrm{Gev} / \mathrm{c}\right)$.

III. High momentum particles $\left(\mathrm{p}_{1 \mathrm{ab}} \gg 20 \mathrm{Gev} / \mathrm{c}\right)$.

The unique feature of our experiment is its ability to achieve comprehensive coverage of regions I and II. Region III could be covered either by the longer configurations of our proposed apparatus or by apparatus already under construction (for experiments 7 and 61) or by the proposed single-arm spectrometer facility.

We therefore propose that we be authorized to measure at an early date particle spectra in regions $I$ and $I I$. These measurements would be carried out with the short, low-momentum configurations of the apparatus described in our proposal. These configurations are modest in scale and use we11-tested detection techniques. Thus we wish to separate the part of our proposal dealing with relatively low laboratory momenta, and we wish to carry out this part first.

In the remainder of this addendum, we discuss our detailed proposals for regions I and II, and various possibilities for region III.

I. Forward particles, $1.5 \leq \mathrm{p}_{1 \mathrm{ab}} \leq 20 \mathrm{Gev} / \mathrm{c}$

This region will be covered by our forward spectrometer as originally proposed, but on $1 y$ in those configurations permitting coverage up to secondary particle laboratory momenta of $20 \mathrm{Gev} / \mathrm{c}$. The total 1ength of the longest $(20 \mathrm{Gev} / \mathrm{c}$ ) configuration is 25 meters. There will be some 
coverage up to $40 \mathrm{Gev} / \mathrm{c}$, at reduced momentum resolution, in order to provide overlap with higher momentum data. Fig. 1 is a drawing of the spectrometer in its shortest configuration. The coverage in terms of Feynman's variable $\mathrm{x}$ brackets the $\mathrm{x}=0$ region. Depending on transverse momentum, and on the mass of particle $c$, the coverage in $x$ extends from a lower 1 imit of from $x=-0.6$ to $x=-0.02$, continuously up to an upper limit of from $x=0.1$ to $x=0.5$. In accordance with our original method of estimating running time, the beam time which we are requesting for this phase is 370 hours (including testing).

II. Backward particles of $\mathrm{p}_{1 \mathrm{ab}} \lesssim 1.5 \mathrm{Gev} / \mathrm{c}$

This range will be covered with our backward spectrometer, as originally proposed. Fig. 2 is drawing of the spectrometer. This spectrometer extends the coverage obtained with the forward spectrometer to $\mathrm{x}=-1.0$

In discussions with the N.A.L. staff, we have determined that there is a rea1 possibility that this phase of the experiment could run in a parasitic mode, upstream of another experiment. Fig. 3 depicts how this can be accomplished, by installing the backward spectrometer in the mezzanine of the Meson Area building. Crane coverage is not required. The target is $10 \mathrm{~cm}$ of liquid hydrogen (volume $\sim 0.1$ liter). The beam requirements could be met by the $2.5 \mathrm{mrad}$ or 3.05 mrad beams, with momenta near 40,80 and $160 \mathrm{Gev} / \mathrm{c}$ and spot size at our target of a few $\mathrm{cm}$. The beam emerges from our experiment undeflected and undisturbed, except for negligible amount of energy loss and multiple scattering. Background produced by our target will be no more than that from counters or other monitors placed in the beam. Of course we are aware of the difficulties, both psychological and logistical, of running experiments in tandem this 
way; however, in view of the possibility of increasing the physics output of N.A.L. at little additional investment, we feel that tandem running in this case is worth a try.

The running time needed for the backward phase is 250 hours. Because of the possibility of tandem running, this phase could be carried out at a very early stage of the N.A.L. research program.

III. High momentum particles $\left(\mathrm{p}_{1 \mathrm{ab}} \geqslant 20 \mathrm{Gev} / \mathrm{c}\right)$.

\section{A. High-Momentum Configurations of our Original Proposal.}

This approach involves an apparatus that is relatively modest in cost, and that is tailored to this specific experiment. Details are given in our proposal.

B. The Strong Focussing Spectrometer Facility.

The device which was discussed at the Single-Arm Spectrometer Workshop would be well suited for studying particle production spectra at large laboratory momenta and smal1 angles.

It was agreed at the workshop that the lower limit of the design range of the spectrometer will be $p_{\min }=20-40 \mathrm{Gev} / \mathrm{c}$. The magnets of course can be tuned lower, but several factors set a useful lower limit for the spectrometer. These are: the maximum accessible scattering. angle of $\sim 80 \mathrm{mrad}$; the problem of decay of mesons over the long path thru the spectrometer; the small acceptance (in $d^{2} \mathrm{p}_{\mathrm{T}}$ ) at low momenta. We had a discussion with Dr. J. Friedman of M.I.T. who is the chairman of the subcommittee that will prepare the proposal for use of the facility in inelastic scattering. It was agreed that our group would not participate in the activities of this subcommittee but that we would keep in touch and, should both efforts be approved, we would try to coordinate the choice of kinematic regions covered and arrange 
for suitable overlap.

C. The Equipment of Experiments 7 and 61 .

It seems quite possible that the forward arm of one of these experiments could be used, essentially unmodified, to obtain single particle distributions at high momenta such as 50,100 and $150 \mathrm{Gev} / \mathrm{c}$. We haye not studied this possibility in detail; in particular we are not sure how one would handle the problem of multiparticle contamination (see below). As far as we know, no one is now actively developing the idea of using the forward arm unchanged.

The remainder of this addendum to our proposal is devoted to a discussion of the multiparticle contamination problem, and to some comments on various features of our experiment. The Multiparticle Contamination Problem.

Multiparticle contamination (see pages 26-27 of our proposal) can be a crucial source of systematic error in a measurement of single particle distributions; it appears that the subtleties of this problem are not universally understood. Imagine a single-arm spectrometer with a Čerenkov counter for particle identification. If the acceptance of the spectrometer is too large, then there will be a significant probability that two particles from a multiparticle event will traverse the Čerenkov counter. (Actually depending on the design, there may be many more particles traversing the counter than are ultimately accepted by the system). Now of course, if there are suitable wire chambers in the spectrometer, one can sort out the trajectories of the two particles. One will then know that two particles were produced in the region of phase space being studied, and one will even know the momentum and angle of each. The problem is that one will now have a class of events without particle identification. Since two-particle correlations may be significant, there 
will thus be a unavoidable systematic error in one's results for the single-particle distributions.

Comments on Our Experimental Design.

Our experiment will have the following key features:

1. Direct control over the multiparticle contamination problem.

2. Constant acceptance in $\mathrm{d}_{\mathrm{P}_{\mathrm{T}}}^{2}$, as large as possible consistent with (1). The solid angle in the $1 \mathrm{ab}$ will vary from $5 \mathrm{msr}$ at $1 \mathrm{Gev} / \mathrm{c}$ to $50 \mu \mathrm{sr}$ at $20 \mathrm{Gev} / \mathrm{c}$.

3. Adequate momentum resolution over the entire range of laboratory momenta covered.

Accuracy. In the most favorable parts of the range of the distributions to be covered, the internal accuracy of the experimental normalization will be a few percent. This capability for high accuracy will be particularly important for studying the dependence of the distributions on incoming energy, and the extent to which they approach high energy limits.

Particle Identification. We believe that data for incident and outgoing $\mathrm{K}^{\prime} \mathrm{s}$ and $\mathrm{p} s \mathrm{can}$ be obtained simultaneously with the data for $\pi^{\prime} \mathrm{s}$ and $\mathrm{p}^{\prime} \mathrm{s}$, at little incremental effort, because of the way in which our apparatus is designed. In particular we do not think that the extra degree of comprehensiveness will in any way degrade the quality of the data for incident and outgoing $\pi^{\prime} s$ and $\mathrm{p}^{\prime} \mathrm{s}$. Should the situation turn out to be otherwise, we shall of course concentrate on the $\pi$ and p part of the experiment on $1 y$.

Spectrometer Motion and Alignment System.

The backward spectrometer is pivoted about the target in a standard way (refer to Fig. 2). 
In the case of the forward configurations, we have chosen the unorthodox technique of transverse motion of individual components. We have found that one reasonable way to achieve the motion is by means of precision slides. These slides, along with their driving motors, and digital electronics, are stock industrial items made for numerical machine control applications. The position accuracy is typically a factor of ten better than we require. The cost is low. Although the techniques of precision mechanical motion are perhaps unfamiliar to some high-energy physicists, we consider this part of our experimental design to be a simple, dependable and elegant solution to a number of experimental problems. (We are still optimizing the motion system, but are confident that we have already achieved a workable solution). The longitudinal motion provided by rails is to be of coarse accuracy only. If suitable crane coverage is available, we need not use rails at all. 


\section{FIGURES}

\section{Figure 1.}

Plan view of the forward spectrometer in its lowest momentum (1.25-2.5 Gev/c) configuration. Secondary particles from the liquid hydrogen target pass through scintillator $S_{1}$, wire chambers $W_{1}, W_{2}$ and $W_{3}$, magnet $M_{1}$ (Pole tip region shown shaded), chambers $W_{4}, W_{5}, W_{6}$, scintillation counter $\mathrm{s}_{2}, \stackrel{v}{ }$ Cerenkov counters $\stackrel{v}{\mathrm{C}}_{1}, \stackrel{v}{\mathrm{C}}_{2}$ and ${\stackrel{v}{\mathrm{C}_{3}}}_{3}$, and chamber $\mathrm{W}_{7}, \mathrm{~A} 12 \mathrm{C} 24$ (P.P.A. or A.G.S.) Magnet is assumed for $M_{1}$. Chambers $W_{3}$ and $W_{4}$ are located at the edges of the $M_{1}$ effective field region, between the $M_{1}$ coils. All components are mounted on precision slides to permit motion transverse to the beam under remote computer control. The slides for $\mathrm{W}_{3}$ and $\mathrm{W}_{4}$ are mounted directly on magnet $\mathrm{M}_{1}$ to permit transverse motion relative to it; this extra motion is necessary in the lowest momentum configurations where the $M_{1}$ field length is an appreciable fraction of the total spectrometer length. The other five slides are mounted on five trolleys which ride on rails, providing longitudinal motion of coarse accuracy. After each configuration change (there are four forward configurations) the precision slides are leveled and aligned with respect to each other and the beam by means of adjusting screws and jacks (not shown) using standard optical surveying techniques. 


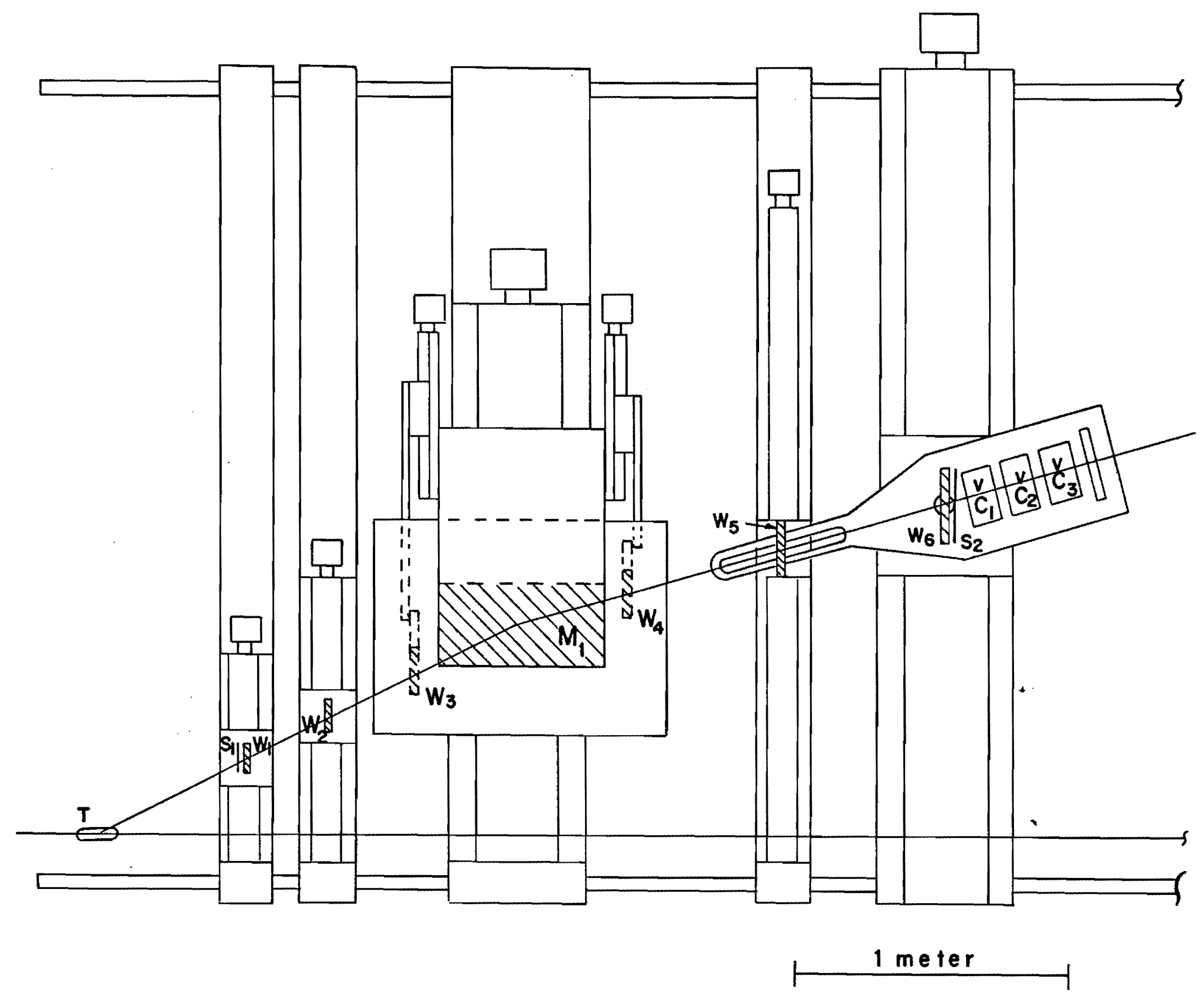




\section{Figure 2.}

Plan view of the backward spectrometer configuration. Secondary particles from the liquid hydrogen target $\mathrm{T}$ pass through scintillator $\mathrm{S}_{1}$, wire chambers $W_{1}, W_{2}$, and $W_{3}$, magnet $M_{1}$, chambers $W_{4}, W_{5}$, and $W_{6}$, scintillation counter $s_{2}$, threshold Cerenkov counters $C_{1}, C_{2}$ and $c_{3}$, and chamber $W_{7}$. A $12 \mathrm{C} 24$ (P.P.A. or A.G.S.) magnet is assumed for $\mathrm{M}_{1}$. The layout differs somewhat from that show in our original proposal in that both the bending plane and the scattering plane are now horizontal, permitting coverage of a wider range of production angles. Chambers $W_{3}$ and $W_{4}$ are located at the edges of the $M_{1}$ effective field region, between the $M_{1}$ coils. A11 components are mounted on a table, which pivots about an axis through the target, and which rides on a circular rail. The production angle viewed by the spectrometer can be changed and read remotely, under computer control. 


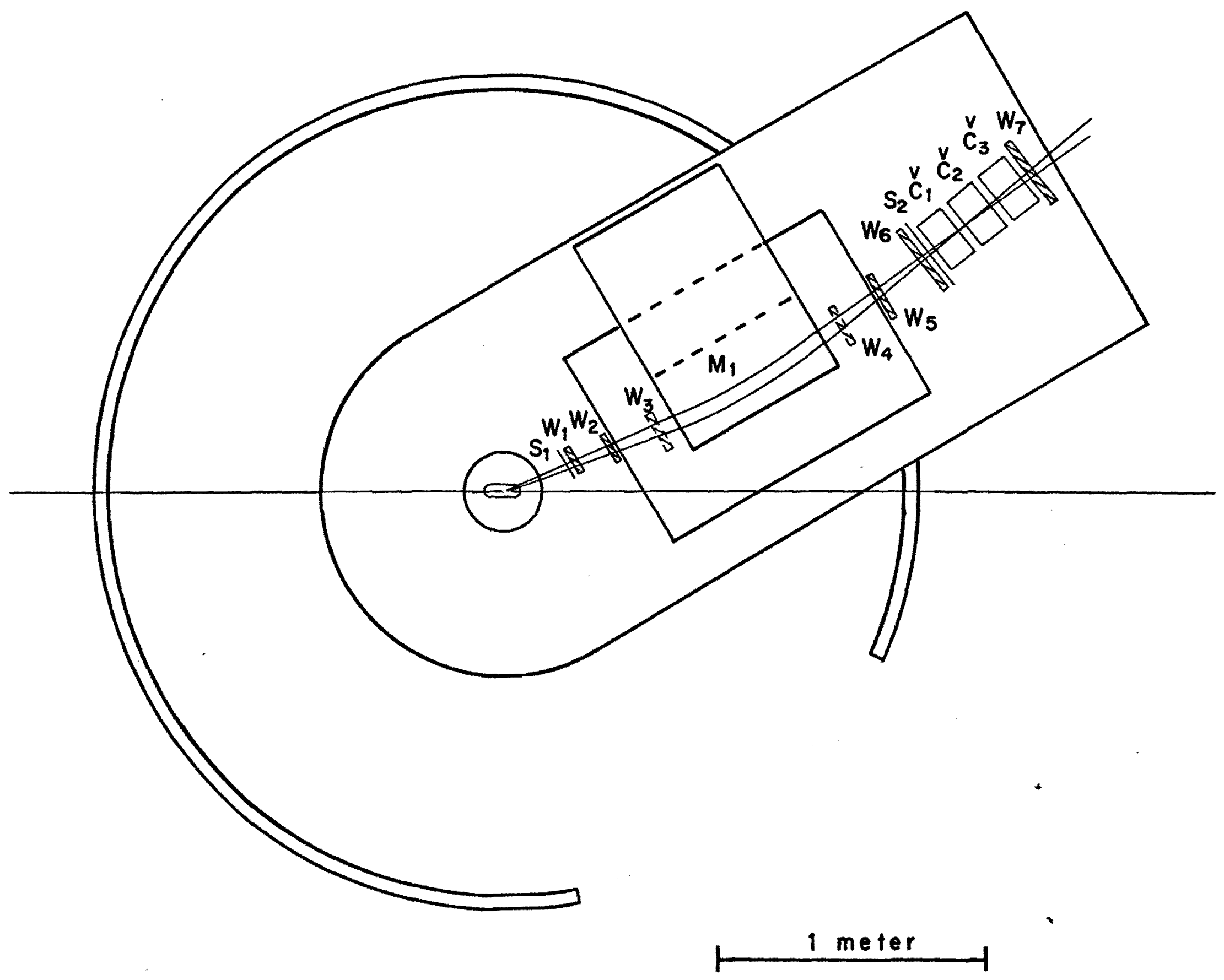




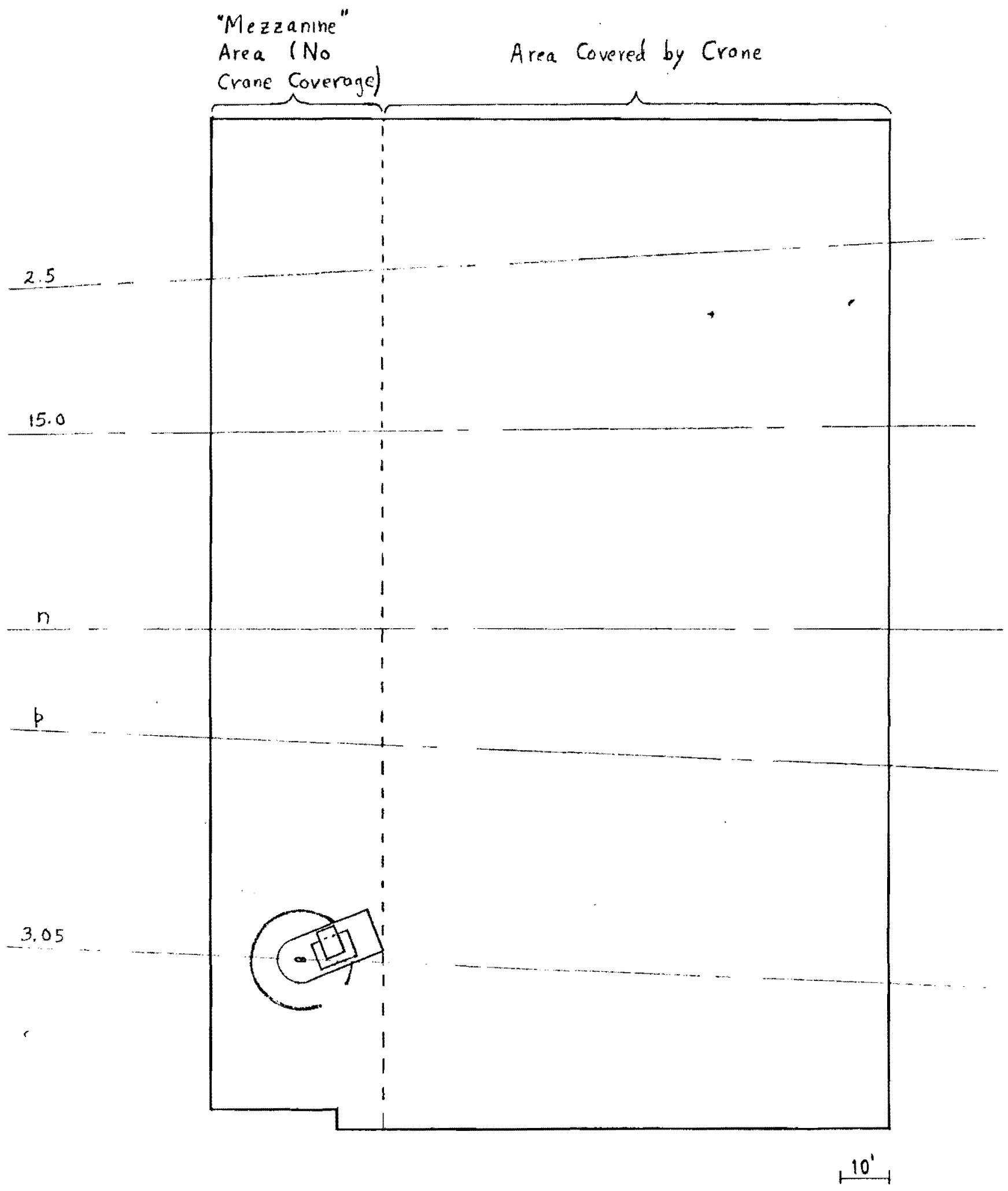

Figure 3.

Plan view of the backward spectrometer installed in the $3.05 \mathrm{mrad}$ beam in the Meson Area, upstream of another experiment. The $2.5 \mathrm{mrad}$ beam will also be suitable. 


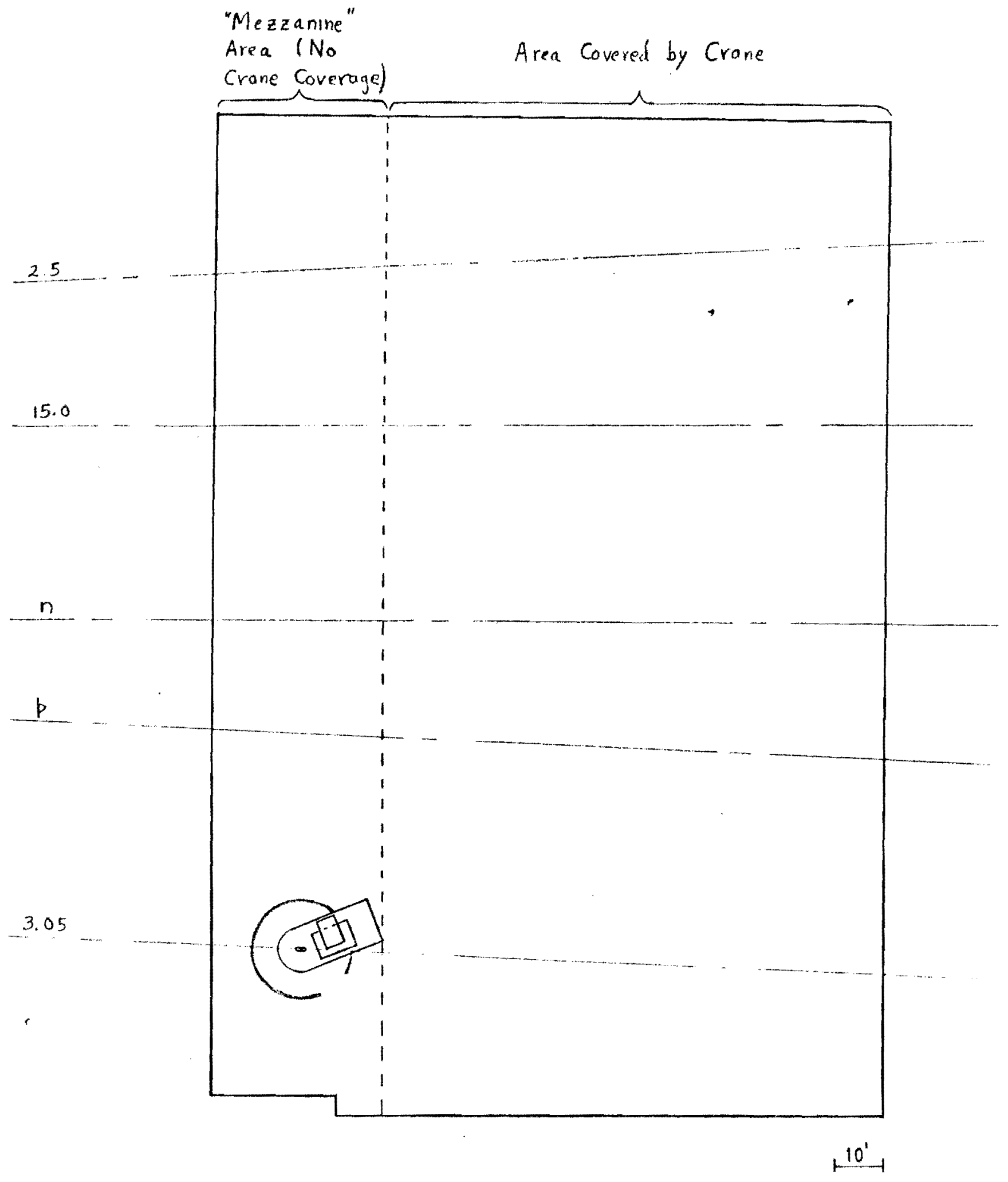

Figure 3.

Plan view of the backward spectrometer installed in the $3.05 \mathrm{mrad}$ beam in the Meson Area, upstream of another experiment. The $2.5 \mathrm{mrad}$ beam will also be suitable. 


\title{
N.A.I. PROPOSAL 52, ADDENDUM 2
}

\author{
Eugene W. Beier, David I. Kreinick, \\ Richard Van Berg and Howard Weisberg \\ University of Pennsylvania \\ July 19, 1971
}

Summary - We propose to make a comprehensive set of measurements of "inclusive scattering" for secondary laboratory momenta up to $20 \mathrm{Gev} / \mathrm{c}$, corresponding to the "target fragmentation" region and the "central" region of secondary momenta. The spectrometer we shall use for these measurements consists of a small C-magnet, Charpak chambers, and threshold Cerenkov counters, on a rigid arm that pivots around a vertical axis thru the target. Our experiment can fit into the "front-porch" area in the $3.5 \mathrm{mrad}$ beam upstream of the Meson Building. The spectrometer is the same one we are constructing for our A.G.S. inclusive scattering experiment which will be run during 1972. The demand on N.A.L. resources will be small. 
Introduction - We have proposed an experiment to measure the differential cross-section for single particle production in the collisions of charged hadrons with protons. Specifically we proposed to measure $\frac{d \sigma}{d^{3} p}$ for the reactions $a+p \rightarrow c+$ anything, where $a, c=\pi^{ \pm}, K^{ \pm}$, and $p^{ \pm}$, over the complete range of secondary momenta. In an addendum dated November 16, 1970 we described how the range of secondary momenta can be divided into various regions.

In the present (and hopefully last:) addendum, we propose specifically to carry out measurements in the region of secondary momenta $P_{\text {lab }}$ $520 \mathrm{Gev} / \mathrm{c}$, corresponding to the "target fragmentation" and "central" region of the kinematic range.

This addendum incorporates some small but important design improvements coming out of work we have done this spring and surmer on the design of the single-arm spectrometer for our A.G.S. experiment on particle production spectra.

Experimental Layout - Figure 1 is a plan view of our A.G.S. layout, which is identical to our proposed N.A.L. layout. There is a ten-foot arm that pivots around the target from 0 to 270 degrees, and an extension to 30 feet that pivots from 0 to 30 degrees. We have found that, by using the "decision making" capability of Charpak chambers, we can cover a wide momentum range with a fixed-length non-focusing spectrometer. The tenfoot length covers the momentum range from 0.3 to $5 \mathrm{Gev} / \mathrm{c}$ in four momentum bands, and the 30-foot length covers 2.5 to $20 \mathrm{Gev} / \mathrm{c}$ in three bands, with some capability also up to $40 \mathrm{Gev} / \mathrm{c}$.

Essentially all of the equipment has been designed and is under 
construction or prototype testing, except that the arm extension and tracks for the 30-foot configuration have been designed but are not under construction.

The 30-foot configuration uses the same spectrometer drive, angle readout, magnet, wire chambers, cables and electronics as the 10 foot one, and uses some of the same Cerenkov counters. We plan to make the configuration changeover once during the course of the experiment. We believe the experiment can fit naturally in the "front-porch" area (total length $40 \mathrm{ft.}$ ) in the $3.5 \mathrm{mrad}$ beam upstream of the Meson Lab. Alternatively, it could go somewhere else in the 2.5 or 3.5 mrad beams.

Performance Parameters - The properties of the two configurations of our spectrometer are given in Table I. These properties are quite similar to those given in our initial proposal, despite the economies of design which have been achieved.

It is of interest to compare these properties with those of a focusing spectrometer, and so the third column shows the properties of the low-momentum focusing spectrometer being built at N.A.L. for use in the proton beam. In the range up to $2.5 \mathrm{Gev} / \mathrm{c}$, the product (solid angle acceptance) $x$ (momentum acceptance) $x$ (target length) is three orders of magnitude greater for our spectrometer. Therefore, for use in secondary beams, the focusing spectrometer is inadequate.

Experimental Coverage and Time Estimate - Figures 2 and 3 are Peyrou plots showing the coverage of our experiment for secondary pions and protons. Each numbered region shown corresponds to a single "sweep" (sequence of 
short runs covering fixed secondary momenta and a sequence of angles.)

Each sweep will be carried out 12 times ( 3 beam momenta $x 2$ primary particle charges $\mathrm{x} 2$ secondary particle charges); some additional running with target empty and some repeats of old settings will also be needed. Table 2 gives, for each sweep, the laboratory momenta and angles covered.

In accordance with the original method of estimating running time given in our proposal, the beam time which we request is a total of 750 hours (including testing).

\section{Beam Requirements -}

1. Momenta: 40,80 and $160 \mathrm{Gev} / \mathrm{c}$.

2. Polarities: Both.

3. Momentum Bite: $\pm 1.0 \%$

4. Angular divergence: $\pm 2.5 \mathrm{mrad}$ or better

5. Spot size: $1 \mathrm{~cm}$ or smaller full width in both planes

6. Halo: Better than $99 \%$ of the hadronic component of the beam should be contained within a $4 \mathrm{~cm}$ diameter circle.

7. Intensity and disposition of the beam downstream of our experiment: Most of our running will be with the maximum available intensity that the Cerenkov counters can handle, and the beam will pass undisturbed thru our apparatus. Our small angle running requires reduced intensity, and the beam may be deflected \pm 2.5 mrad by our spectrometer magnet. 
Equipment Requirements -

1. Experimental area: Layout is shown in Figure 1. An enclosed area for housing the electronics should be provided by N.A.L.

2. Liquid hydrogen target: Standard target with 8 inch long, 2 inch diameter flask. We no longer require remote changing from full to empty target.

3. Spectrometer magnet: One 12C24 magnet. Its power supply should have low ripple to prevent vibrations in the Charpak chambers which are placed in the magnet fringe field.

4. Spectrometer mounting and alignment system: To be furnished entirely by us. If there is no crane coverage, then a fork-lift truck is needed for initial installation of the tracks and magnet, and once during the experiment to move the magnet for the configuration changeover.

5. Beam instrumentation: To be provided by N.A.L. for simultaneous identification of pions, kaons and protons.

6. Computer facilities: We no longer are assured that the University of Pennsylvania PDP-9 computer, which we shall be using at the A.G.S., will be available for N.A.L. use. Therefore we request that N.A.L. provide a PDP-15, PDP-II or similar computer with at least $8 \mathrm{~K}$ of memory, a high-speed tape drive, a CRT display, and a CAMAC interface. We also require either an on-line link or else fast turn-around (a few hours) off-line access to a large floating-point computer.

Manpower - The experiment will be manned by physicists from the University of Pennsylvania. 
Schedule - Our A.G.S. experiment is scheduled to go on the floor in February 1972, and to be finished by the end of 1972 . This schedule has not slipped at all since last December and we expect that it will be met. Therefore, we propose being installed at N.A.I. in January, 1973. 
SPECTROMETER CHARACTERISTICS

\begin{tabular}{|c|c|c|c|}
\hline & \multicolumn{2}{|c|}{ This Proposal } & \multirow{2}{*}{$\begin{array}{l}\text { N.A.L.- } \\
\text { Walker* }\end{array}$} \\
\hline & $\begin{array}{c}\text { Short } \\
\text { Configuration } \\
\end{array}$ & $\begin{array}{c}\text { Long } \\
\text { Configuration } \\
\end{array}$ & \\
\hline Type & $\begin{array}{c}\text { Non-Focusing } \\
\text { Rotatable }\end{array}$ & $\begin{array}{l}\text { Non-Focusing, } \\
\text { Rotatable }\end{array}$ & $\begin{array}{l}\text { Focusing, } \\
\text { Rotatable }\end{array}$ \\
\hline Length, ft. & 10 & 30 & 24 \\
\hline $\mathrm{P}_{\max }, \mathrm{Gev} / \mathrm{c}$ & 5 & 20 & $2-2 \cdot 5$ \\
\hline$\theta_{\text {min }}$, degrees & $6-12 * *$ & $2-4 * *$ & 5 \\
\hline$\theta_{\text {max }}$, degrees & 180 & 30 & 175 \\
\hline$\Delta \mathrm{P} / \mathrm{P}_{\text {central }}$ & $75 \%$ & $75 \%$ & $10 \%$ \\
\hline$\delta_{p}, \%$ & $0.4-1.0 \mathrm{r.m} . \mathrm{s}$ & $0.4-0.8 \mathrm{r.m.s}$. & \pm 1.2 \\
\hline $\mathrm{L}_{\text {target }} \mathrm{cm}$ & 10 & 10 & 1 \\
\hline$\Delta \Omega, \mathrm{sr}$ & $5 \times 10^{-3}$ & $0.5 \times 10^{-3}$ & $0.2 \times 10^{-3}$ \\
\hline
\end{tabular}

* Figures given by J. K. Walker at 1971 N.A.L. Users' Meeting.

** Limited by multiparticle contamination. 
KINEMATIC REGIONS COVERED*

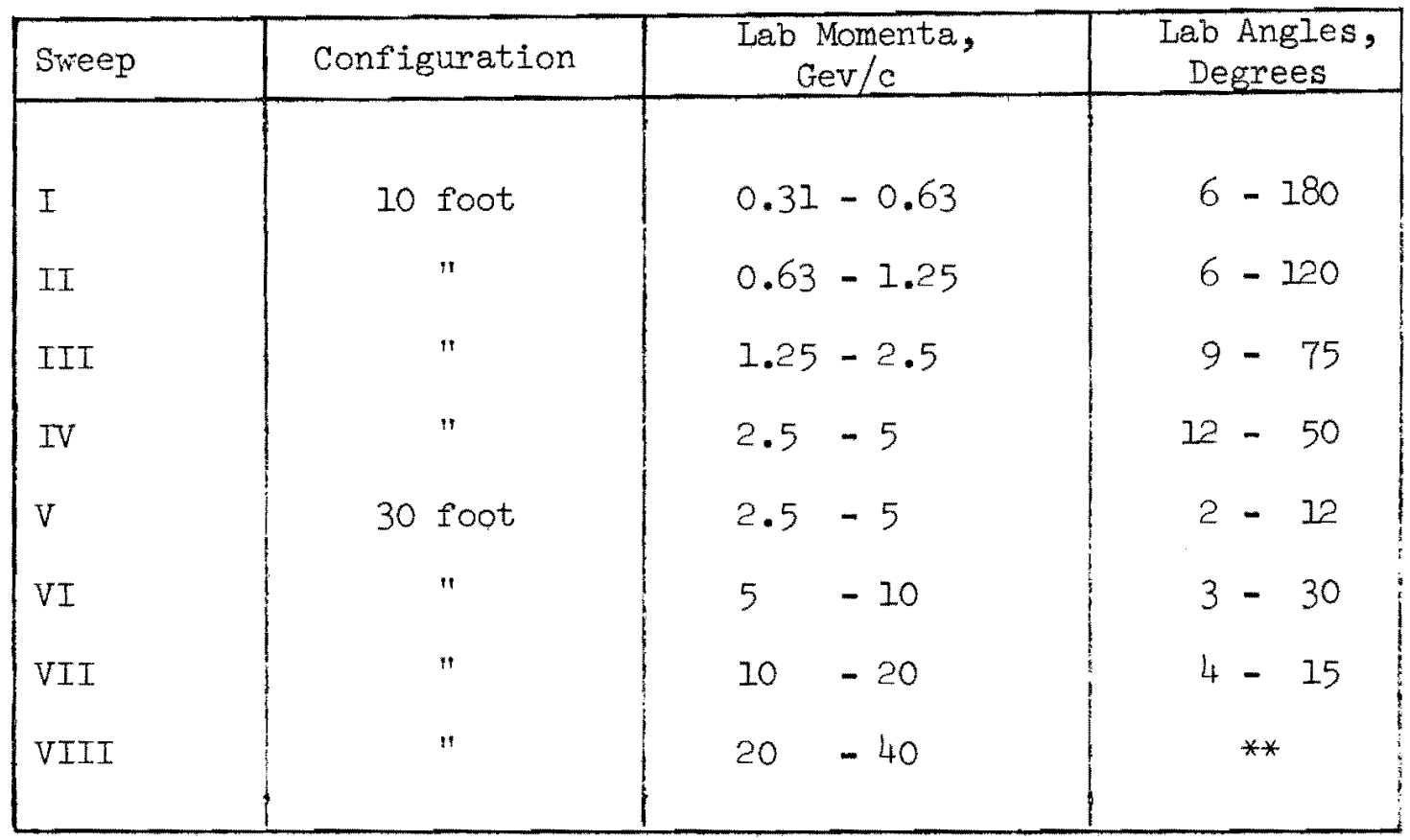

* The actual range of momenta and angles covered in each sweep will be somewhat larger than shown, to allow for overlap.

* Some running can be done at $20-40 \mathrm{Gev} / \mathrm{c}$ to provide overlap with other experiments. 


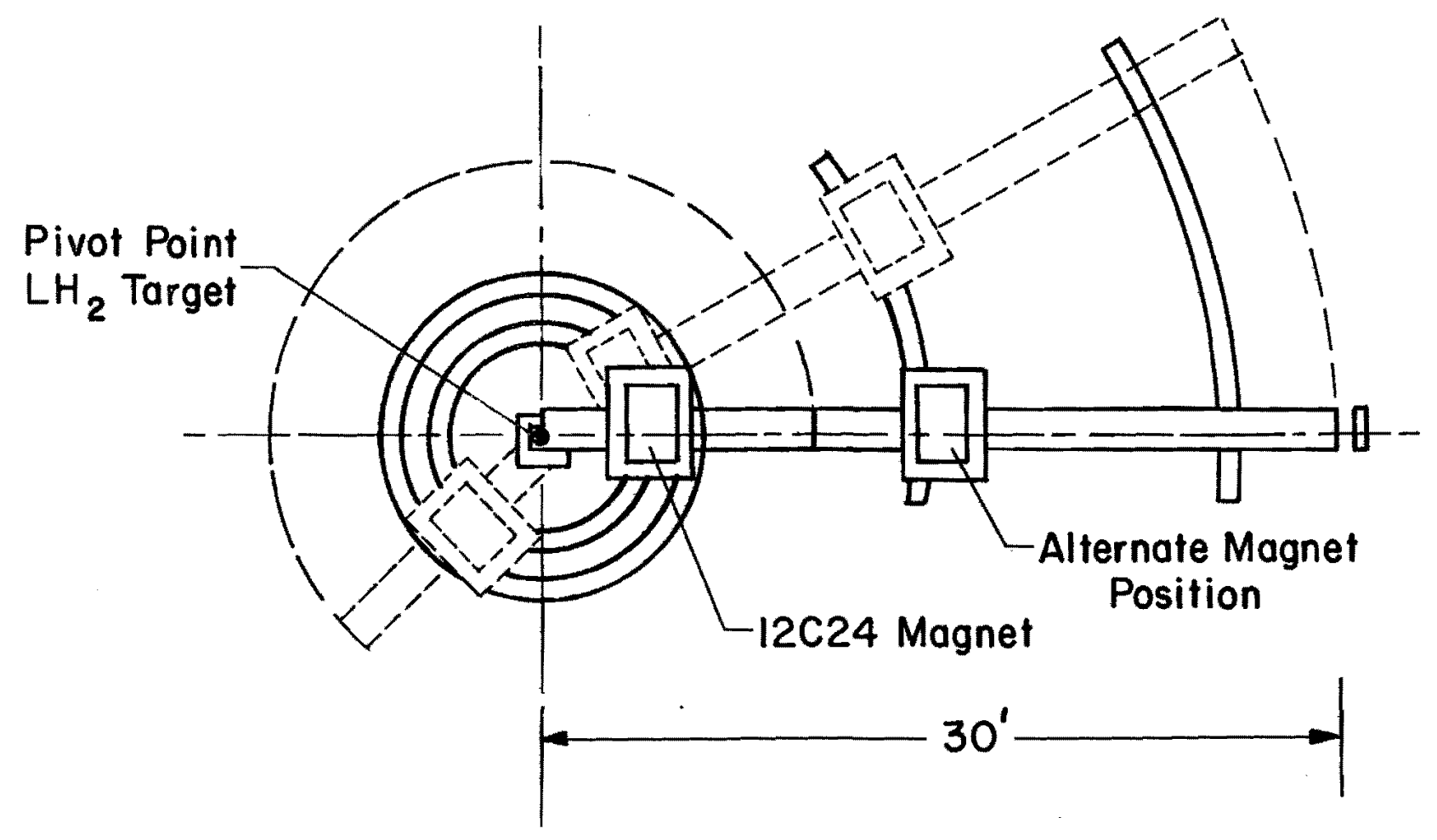

Figure 1. Overall layout of the experiment. 


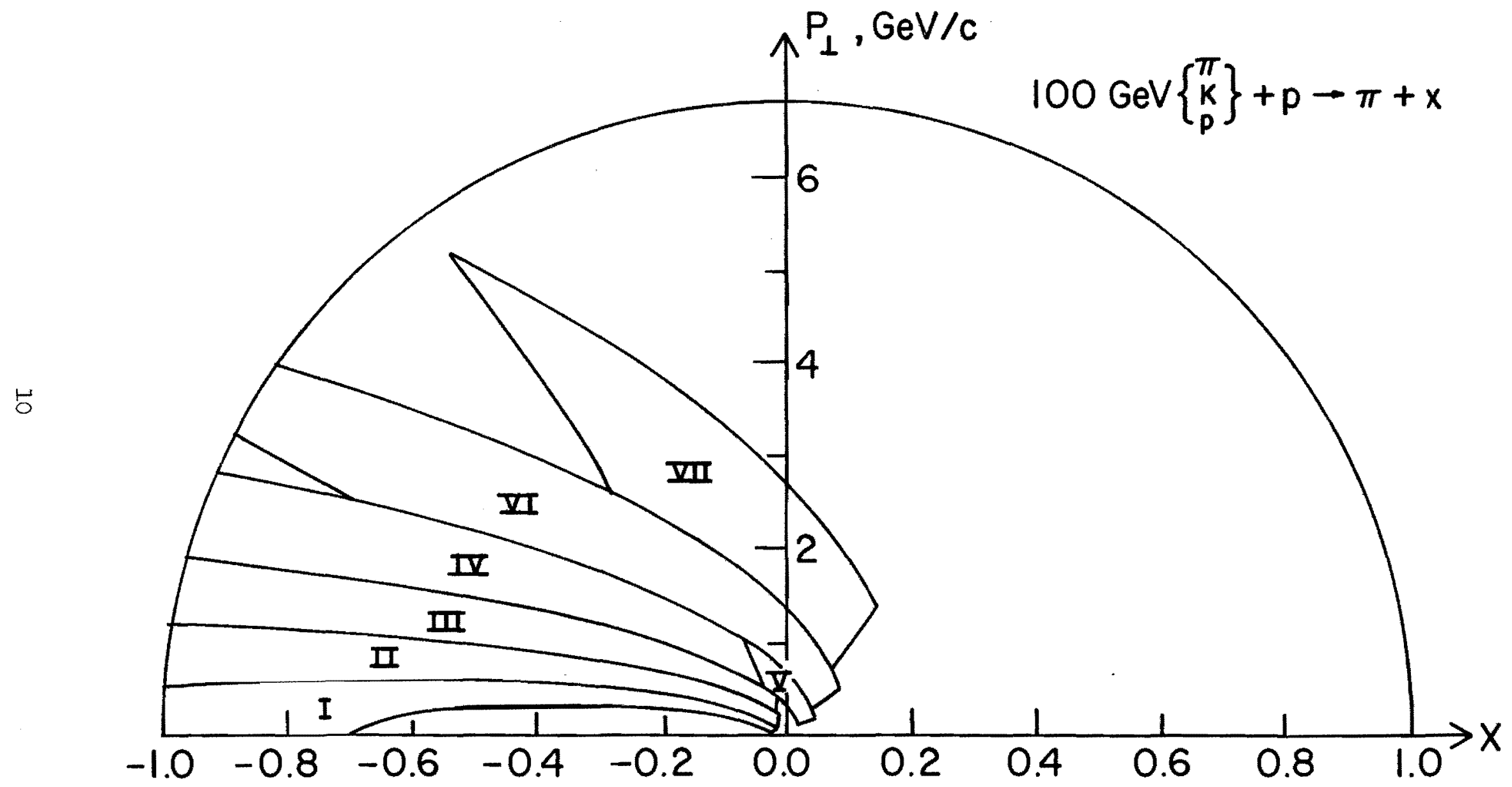

Figure 2. Peyrou plot showing the kinematic region covered in our experiment for the production of pions by $100 \mathrm{Gev}$ pions, kaons or protons. 


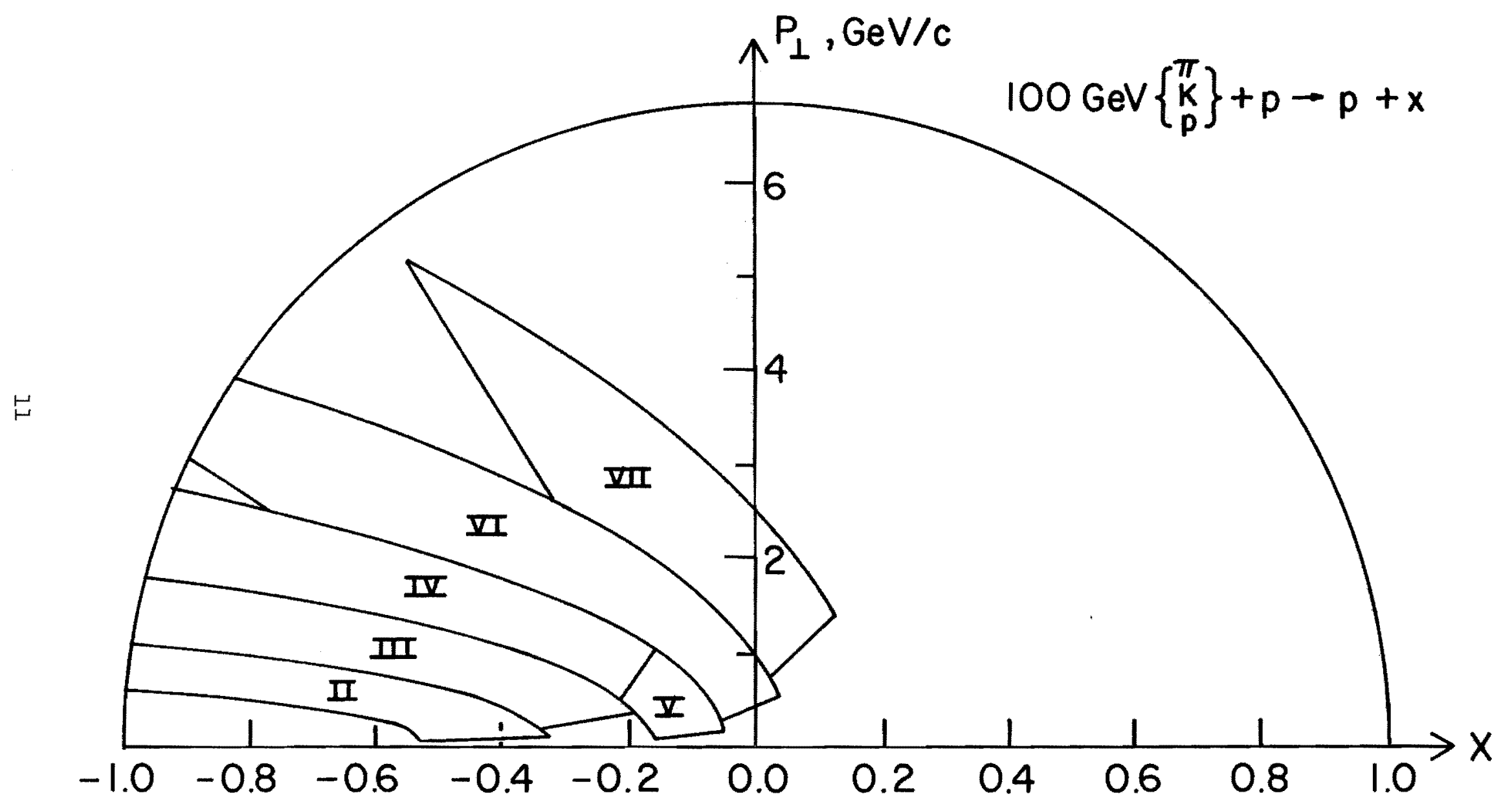

Figure 3. Peyrou plot showing the kinematic region covered in our experiment for the production of protons by $100 \mathrm{Gev}$ pions, kaons or protons. 